\title{
CONCENTRATION ALONG GEODESICS FOR A NONLINEAR STEKLOV PROBLEM ARISING IN CORROSION MODELING*
}

\author{
CARLO D. PAGANI ${ }^{\dagger}$, DARIO PIEROTTI ${ }^{\ddagger}$, ANGELA PISTOIA $^{\S}$, AND GIUSI VAIRA ${ }^{\S}$
}

\begin{abstract}
We consider the problem of finding pairs $(\lambda, \mathfrak{u})$, with $\lambda>0$ and $\mathfrak{u}$ a harmonic function in a three-dimensional torus-like domain $\mathcal{D}$, satisfying the nonlinear boundary condition $\partial_{\nu} \mathfrak{u}=\lambda \sinh \mathfrak{u}$ on $\partial \mathcal{D}$. This type of boundary condition arises in corrosion modeling (Butler-Volmer condition). We prove the existence of solutions which concentrate along some geodesics of the boundary $\partial \mathcal{D}$ as the parameter $\lambda$ goes to zero.
\end{abstract}

Key words. Steklov problem, concentration along geodesics, corrosion modeling

AMS subject classifications. Primary, 35J65; Secondary, 35J05, 58E05

DOI. $10.1137 / 15 \mathrm{M} 1027024$

1. Statement of the problem and main results. In mathematical modeling of electrochemical corrosion a suitably defined galvanic potential satisfies an elliptic equation (namely, the Laplace or Poisson equation in the simplest cases) in a given domain $\mathcal{D}$, whose boundary is partly electrochemically active and partly inert. In the inactive boundary region, the current density flow is of course zero, but in the active part it is modeled (by interpolating experimental data) by a difference of two exponentials according to the so-called Butler-Volmer formula (see [12] for a detailed discussion of the model). Then the resulting mathematical problem consists of solving an elliptic equation complemented with a boundary condition of Neumann type, namely

$$
\partial_{n} \mathfrak{u}(y)=\lambda \mu(y)\left[e^{\alpha \mathfrak{u}(y)}-e^{-(1-\alpha) \mathfrak{u}(y)}\right], \quad y \in \partial \mathcal{D} .
$$

Here $n$ is the outward unit normal to $\partial \mathcal{D}, \mathfrak{u}$ is the surface potential, $\alpha \in(0,1)$ is a constant depending on the constituents of the electrochemical system, $\mu(y)$ is a nonnegative bounded function which distinguishes between the active and the inert boundary regions (typically $\mu(y)$ is the characteristic function of some subset of $\partial \mathcal{D}$ ), and $\lambda$ is a positive parameter.

Due to the exponential growth of the nonlinear boundary term, this problem has been studied (usually for Laplace or Poisson equations) in two dimensions; see [12], $[6],[7],[8]$.

In the physically relevant three-dimensional case, little is known about existence and properties of solutions (see [9], [10]).

A case which presents some interest for applications (such as, for instance, corrosion of sections of pipelines, rings, and bolts) arises when the body $\mathcal{D}$ is a threedimensional annular shaped domain, namely it can be represented in the form

$$
\mathcal{D}=\left\{\left(y_{1}, y_{2}, y_{3}\right) \in \mathbb{R}^{3} \mid\left(\sqrt{y_{1}^{2}+y_{2}^{2}}, y_{3}\right) \in \Omega\right\}
$$

\footnotetext{
*Received by the editors June 22, 2015; accepted for publication (in revised form) December 22, 2015; published electronically March 17, 2016.

http://www.siam.org/journals/sima/48-2/M102702.html

$\dagger$ Istituto Lombardo, 20121 Milano, Italy (carlo.pagani@polimi.it).

${ }^{\ddagger}$ Dipartimento di Matematica, Politecnico di Milano, 20133 Milano, Italy (dario.pierotti@polimi.

$\S$ Dipartimento di Scienze di Base e Applicate per l'Ingegneria, Sapienza Università di Roma, 00161 Roma, Italy (angela.pistoia@uniroma1.it, vaira.giusi@gmail.com).
} it).

1085 
and $\Omega$ is a smooth bounded domain in $\mathbb{R}^{2}$ such that

$$
\bar{\Omega} \subset\left\{\left(x_{1}, x_{2}\right) \in \mathbb{R}^{2} \mid x_{1}>0\right\} .
$$

Clearly, the domain $\mathcal{D}$ is $\mathcal{G}$-invariant for the action of the group $\mathcal{G}$ given by

$$
g\left(y_{1}, y_{2}, y_{3}\right)=\left(\tilde{g}\left(y_{1}, y_{2}\right), y_{3}\right),
$$

where $\tilde{g} \in \mathcal{O}(2)$ (the group of linear isometries of $\mathbb{R}^{2}$ ).

The chosen geometry aims at modelizing the corrosion of torus-like bodies. Then, we consider the boundary value problem

$$
\left\{\begin{array}{l}
\Delta \mathfrak{u}=0 \quad \text { in } \mathcal{D}, \\
\partial_{n} \mathfrak{u}=\lambda \sinh \mathfrak{u} \quad \text { on } \partial \mathcal{D} .
\end{array}\right.
$$

Note that we have chosen in (1.1) $\alpha=1 / 2, \mu \equiv 1$, and we wrote $\mathfrak{u}$ instead of $\mathfrak{u} / 2$ for the harmonic potential (existence results for the problem with $\alpha \neq 1 / 2$ and $\mu$ not identically equal to 1 are discussed in [7]).

In such a situation it is natural to look for solutions which are $\mathcal{G}$-invariant; i.e., they are axially symmetric functions of the form

$$
\mathfrak{u}\left(y_{1}, y_{2}, y_{3}\right)=u\left(x_{1}, x_{2}\right),
$$

where $x_{1}=\left(y_{1}^{2}+y_{2}^{2}\right)^{1 / 2}$ and $x_{2}=y_{3}$.

Now an easy computation shows that (1.4) is equivalent to the following problem for $u$ :

$$
\begin{cases}\operatorname{div}\left(x_{1} \nabla u\right)=0 & \text { in } \Omega, \\ \partial_{\nu} u=\lambda \sinh u & \text { on } \partial \Omega .\end{cases}
$$

Thus, we are led to study the more general anisotropic two-dimensional problem

$$
\left\{\begin{array}{l}
\Delta_{a} u=0 \text { in } \Omega, \\
\partial_{\nu} u=\lambda \sinh u \text { on } \partial \Omega
\end{array}\right.
$$

with $a \in L^{\infty}(\Omega)$ such that

$$
0<a_{0} \leq a\left(x_{1}, x_{2}\right) \leq a_{1}<\infty \quad \text { a.e. in } \Omega,
$$

$\lambda$ is a positive parameter, and $\Delta_{a} u$ is defined as

$$
\Delta_{a} u=\frac{1}{a(x)} \operatorname{div}(a(x) \nabla u)=\Delta u+\nabla \log a(x) \cdot \nabla u
$$

for every $u \in H^{1}(\Omega)$.

We remark that problem (1.6) corresponds to choosing $a\left(x_{1}, x_{2}\right) \equiv x_{1}$.

The main goal of the present paper is to extend to the anisotropic problem (1.7) the principal results obtained for $a \equiv 1$ concerning both existence of multiple solutions and the limiting behavior of the solutions for $\lambda \rightarrow 0^{+}$.

In what follows we first (section 2) prove existence of global multiple solutions of problem (1.7) and, as a consequence, of problem (1.4). This is done by a mild modification of the variational approach used in [8]. We prove the following result.

Theorem 1.1. Let $\Omega \subset \mathbb{R}^{2}$ be a Lipschitz domain and let $a \in L^{\infty}(\Omega)$ such that (1.8) is satisfied. Then for every $\lambda>0$ there exist infinitely many solutions (in $H^{1}(\Omega)$ ) of problem (1.7). 
Then, a simple corollary states the existence of solutions of problem (1.4).

COROLlary 1.2. For every $\lambda>0$ there are infinitely many axially symmetric solutions to problem (1.4).

Moreover, in subsection 2.3 a first approach to the description of the behavior of the solutions for $\lambda \rightarrow 0^{+}$is considered. For $a \equiv 1$ this study was done in [6]; there the authors prove, for a bounded $\mathcal{C}^{2, \alpha}$ domain $\Omega \subset \mathbb{R}^{2}$, that all solutions have energies that are of order $\log (1 / \lambda)$; the limit of the boundary flux along subsequences is a sum of Dirac masses located at a finite set of points, potentially accompanied by a regular part of definite sign. These results are a generalization of those obtained in [2] by studying the behavior of explicit solutions of the problem in the unit disk. We show that the same kinds of results hold for the anisotropic case, and we sketch the main arguments of the proofs.

In [5], considering again the case $a \equiv 1$, the authors prove that in any smooth domain $\Omega$ there are at least two distinct families of solutions which exhibit exactly the qualitative behavior of the explicit solutions found in [2], namely with limiting boundary flux given by an array of Dirac masses with weight $2 \pi$ and alternate signs. We will show in section 3, that, for the anisotropic problem (1.7), the situation is substantially the same as depicted in [5]. In that case the arguments used to extend the results of [5] to the anisotropic case are in some part different from those given in [5].

In order to state our result let $x=\left(x_{1}, x_{2}\right) \in \Omega, y=\left(y_{1}, y_{2}\right) \in \partial \Omega$, and let $G_{a}(x, y)$ be the Green's function for the Neumann problem

$$
\left\{\begin{array}{l}
-\left(\Delta_{a}\right)_{x} G_{a}(x, y)=0 \quad \text { in } \Omega, \\
\frac{\partial G_{a}}{\partial \nu_{x}}=2 \pi \delta_{y}(x)-\frac{2 \pi}{\int_{\partial \Omega} a(x)} a(y) \quad \text { on } \partial \Omega, \\
\int_{\partial \Omega} G_{a}(x, y)=0,
\end{array}\right.
$$

and define $H_{a}(x, y)$ as the regular part of $G_{a}(x, y)$, namely

$$
H_{a}(x, y)=G_{a}(x, y)-\log \frac{1}{|x-y|^{2}} .
$$

We say that $\xi \in \partial \Omega$ is a $C^{1}$-stable critical point of $\left.a\right|_{\partial \Omega}$ if $\nabla_{\partial \Omega} a(\xi)=0$ and the local Brouwer degree $\operatorname{deg}\left(\nabla_{\partial \Omega} a, B(\xi, \rho) \cap \partial \Omega, 0\right)$ is well defined (for $\rho$ small enough) and it is not zero. It is easy to see that any strict local minimum point or strict local maximum point or nondegenerate critical point of $\left.a\right|_{\partial \Omega}$ is a $C^{1}$-stable critical point.

Now, the main result is the following theorem.

TheOREM 1.3. Let $\Omega \subset \mathbb{R}^{2}$ be a $C^{1}$-domain and $a \in C^{1}(\bar{\Omega})$. Let $\xi_{1}, \xi_{2} \in \partial \Omega$ be two different $C^{1}$-stable critical points of a $\left.\right|_{\partial \Omega}$. Then there is $\lambda_{0}>0$ such that, for $0<\lambda<\lambda_{0}$, there is a sign-changing solution $u_{\lambda}$ of the problem (1.7) of the form

$$
u_{\lambda}(x)=\log \frac{2 \mu_{1}}{\left|x-\left(\xi_{1}+\lambda \mu_{1} \nu_{1}\right)\right|^{2}}-\log \frac{2 \mu_{2}}{\left|x-\left(\xi_{2}+\lambda \mu_{2} \nu_{2}\right)\right|^{2}}+O(1),
$$

where $\nu_{1}$ and $\nu_{2}$ denote the unit outer normals to $\partial \Omega$ at the points $\xi_{1}$ and $\xi_{2}$, respectively, and the parameters $\mu_{1}$ and $\mu_{2}$ are explicitly given by

$$
\mu_{1}=\frac{1}{2} e^{H_{a}\left(\xi_{1}, \xi_{1}\right)-G_{a}\left(\xi_{1}, \xi_{2}\right)}, \quad \mu_{2}=\frac{1}{2} e^{H_{a}\left(\xi_{2}, \xi_{2}\right)-G_{a}\left(\xi_{2}, \xi_{1}\right)} .
$$


In particular, the solution $u_{\lambda}$ concentrates positively and negatively at the points $\xi_{1}$ and $\xi_{2}$, respectively, as $\lambda$ goes to zero.

According to the previous discussion, the corresponding result for problem (1.4) reads as follows.

Corollary 1.4. Let $\Omega \subset \mathbb{R}^{2}$ be a $C^{1}$-domain and $a \in C^{1}(\bar{\Omega})$. Let $\xi_{1}, \xi_{2} \in \partial \Omega$ be two different $C^{1}$-stable critical points of $a(x)=x_{1}$ restricted on $\partial \Omega$. Then there exists $\lambda_{0}>0$ such that for any $\lambda \in\left(0, \lambda_{0}\right)$, problem (1.4) has a sign-changing axially symmetric solution $\mathfrak{u}_{\lambda}$ which concentrates positively and negatively along two geodesics of the boundary $\partial \mathcal{D}$ which are the $\mathcal{G}$-orbits of $\xi_{1}$ and $\xi_{2}$, respectively, as $\lambda$ goes to zero.

The proof of Theorem 1.3 relies on a very well-known finite dimensional procedure, and it is carried out in section 3. We shall omit many details of this proof because they can be found up to minor modifications in [5], where problem (1.7) has been studied with $a(x) \equiv 1$. We only compute what cannot be deduced from known results.

We point out that in our case the anisotropic term $a$ plays an essential role in the construction of our solutions. Indeed the location of the concentration points of the solutions is completely determined by the critical points of $a$ itself. That is why we need to carry out a careful analysis of the regular part of the Green's function defined in (1.11) associated with a linear anisotropic operator depending on $a$ (see Lemmas 3.1 and 3.2 ).

Our result suggests that it should be possible to find solutions which concentrate along two geodesics of the boundary of a more general torus-like three-dimensional domain which is not necessarily axially symmetric.

2. Existence and multiplicity of solutions. The content of this section almost repeats the arguments developed in [8]: recall that we have chosen the weight function $\mu(x)$ appearing in (1.1) identically 1, and that we deal with the operator $\Delta_{a}$ instead of the Laplacian; therefore, many statements are simple rephrasings of the corresponding statements given in [8] and are not given here in full detail. Let us first recall that the approach to the nonlinear problem relies on the solution of a related linear Steklov eigenvalue problem on the boundary. We summarize here (without proofs) the crucial results about this problem.

Let $\Omega \subset \mathbb{R}^{N}$ be a bounded Lipschitz domain and consider the following linear Steklov eigenvalue problem in $H^{1}(\Omega)$ :

$$
\left\{\begin{array}{l}
\operatorname{div}(a(x) \nabla u)=0 \quad \text { in } \Omega, \\
\partial_{\nu} u=\lambda u \text { on } \partial \Omega .
\end{array}\right.
$$

It is easily seen that for $\lambda>0$ the solutions to (2.1) belong to the subspace $H_{a}^{1} \subset$ $H^{1}(\Omega)$ defined as follows:

$$
H_{a}^{1} \equiv\left\{u \in H^{1}(\Omega), \quad \int_{\partial \Omega} a u=0\right\} .
$$

It can be shown (by a classical reductio ad absurdum argument; see, e.g., [7]) that in $H_{a}^{1}$ the Dirichlet norm $\int_{\Omega}|\nabla u|^{2}$ is equivalent to the $H^{1}$ norm and that (2.1) is equivalent to the following variational problem.

Find $u \in H_{a}^{1}, u \neq 0$, such that

$$
\int_{\Omega} a \nabla u \nabla \varphi=\lambda \int_{\partial \Omega} a u \varphi
$$


holds for every $\varphi \in H^{1}$. Furthermore, the expression

$$
\|u\|_{a}^{2}=\int_{\Omega} a|\nabla u|^{2}+\left(\int_{\partial \Omega} a u\right)^{2}
$$

defines an equivalent norm in $H^{1}(\Omega)$. We will consider the scalar product in $H^{1}(\Omega)$ associated to this equivalent norm; by a slight adaptation of the proof in $[7$, Theorem 2.1 , we have the following proposition.

Proposition 2.1. Problem (2.3) has infinitely many eigenvalues

$$
0<\lambda_{1} \leq \lambda_{2} \leq \cdots
$$

each of finite multiplicity and such that $\left|\lambda_{n}\right| \rightarrow+\infty$. The eigenvalue $\lambda_{0}=0$ corresponds to the constant solutions of the homogeneous Neumann problem. Moreover, we can take all the eigenfunctions $v_{n}, n \geq 0$, orthogonal and normalized with respect to the scalar product associated to the equivalent norm (2.4), and the following decomposition holds:

$$
H^{1}=H_{0}^{1} \oplus V_{a},
$$

where the subspace $V_{a}$ is spanned by the eigenfunctions $v_{n}$ (note that $V_{a}$ contains the subspace of the constant functions).

Remark 2.2. Global regularity of the eigenfunctions depends on the weight $a(x)$ and on the regularity of the boundary $\partial \Omega$. It can be shown that on a Lipschitz domain $\Omega$ the eigenfunctions are bounded and continuous on $\bar{\Omega}$ (see [8], [9], and references therein).

2.1. Main estimates. Let us consider now the nonlinear problem (1.7); we define the even functional

$$
E_{\lambda}(u)=\frac{1}{2} \int_{\Omega} a|\nabla u|^{2}-\lambda \int_{\partial \Omega} a(\cosh u-1),
$$

where $u \in H^{1}(\Omega)$. Note that

$$
\cosh u-1=\frac{u^{2}}{2}+u^{4} h(u),
$$

where, for every $q>1$,

$$
h: H^{1}(\Omega) \rightarrow L^{q}(\partial \Omega)
$$

is bounded (see [6, Lemma 2.1]).

Define further

$$
S_{R}=\left\{u \in H^{1}:\|u\|_{a}=R\right\},
$$

where \|\|$_{a}$ is the equivalent norm defined in (2.4). Then, we have the following lemma.

LEMma 2.3. For every $\lambda>0$, there exist $R>0$ and a closed subspace $V^{+} \subseteq$ $H^{1}(\Omega)$ with codim $V^{+}<\infty$ such that

$$
E_{\lambda}(u) \geq c_{0}>0
$$

for every $u \in S_{R} \cap V^{+}$.

Copyright (c) by SIAM. Unauthorized reproduction of this article is prohibited. 
Proof. By (2.7) we have

$$
E_{\lambda}(u) \geq \frac{1}{2} \int_{\Omega} a|\nabla u|^{2}-\frac{\lambda}{2} \int_{\partial \Omega} a u^{2}-\lambda \int_{\partial \Omega} a|u|^{4} h(u),
$$

and for every $q>1, p=q /(q-1)$, the integral in the last term can be bounded as follows:

$$
\left.\left|\int_{\partial \Omega} a\right| u\right|^{4} h(u) \mid \leq\|a\|_{L^{\infty}(\partial \Omega)}\|h(u)\|_{L^{q}(\partial \Omega)}\left(\int_{\partial \Omega}|u|^{4 p}\right)^{1 / p} \leq C\|u\|_{a}^{4}=C R^{4} .
$$

Let us now consider the quadratic part of the functional. Since the sequence of positive eigenvalues $\lambda_{n}$ of the linear problem is unbounded, there exist nonnegative integers $k, r$, such that

$$
\lambda_{k} \leq \lambda<\lambda_{k+r}
$$

Then, we set

$$
V^{+}=H_{0}^{1} \oplus \operatorname{span}_{n \geq k+r}\left\{v_{n}\right\} .
$$

For every $u \in S_{R} \cap V^{+}$we get

$$
\frac{1}{2} \int_{\Omega} a|\nabla u|^{2}-\frac{\lambda}{2} \int_{\partial \Omega} a u^{2} \geq \frac{1}{2}\left(1-\frac{\lambda}{\lambda_{k+r}}\right) \int_{\Omega} a|\nabla u|^{2}=\frac{1}{2}\left(1-\frac{\lambda}{\lambda_{k+r}}\right) R^{2} .
$$

Then, the result follows by taking $R$ small enough.

We are now going to construct closed, finite dimensional subspaces, $V^{-} \subset H^{1}(\Omega)$ such that

- $\operatorname{dim} V^{-}>\operatorname{codim} V^{+}$;

- $E_{\lambda}(u) \leq c_{\infty}<\infty$ for every $u \in V^{-}$.

To this aim, let $v_{n_{i}}, 1 \leq i \leq l$, be any finite sequence of $l$ eigenfunctions, with $l>\operatorname{codim} V^{+}$, corresponding to the eigenvalues

$$
\lambda_{n_{1}} \leq \lambda_{n_{2}} \leq \cdots \leq \lambda_{n_{l}} .
$$

Let us define

$$
V^{-}=\operatorname{span}_{1 \leq i \leq l}\left\{v_{n_{i}}\right\} .
$$

The next lemma provides the key estimates at infinity on the functional (2.6).

Lemma 2.4. Let $V^{-}$be defined by (2.15). Then, for every $\lambda>0$ we have $E_{\lambda}(u)<0$ for any $u \in V^{-}$with large enough norm. As a consequence, there exists $c_{\infty}<\infty$ such that

$$
E_{\lambda}(u) \leq c_{\infty} \quad \forall u \in V^{-} .
$$

Proof. We first assume $\lambda_{n_{1}}>0$. For notational simplicity, from now on we set $v_{n_{i}}=v_{i}, \lambda_{n_{i}}=\lambda_{i}>0(1 \leq i \leq l)$. Thus, we can write any $u \in V^{-}$in the form

$$
u=\sum_{i=1}^{l} t_{i} v_{i}
$$

Copyright $@$ by SIAM. Unauthorized reproduction of this article is prohibited. 
Recalling Remark 2.2, $u$ is a bounded continuous function on $\Omega$, so that $\sinh u \in$ $H^{1}(\Omega)$ and by Proposition 2.1 the variational equation

$$
\int_{\Omega} a \nabla v_{j} \nabla \sinh u=\lambda_{j} \int_{\partial \Omega} a v_{j} \sinh u
$$

holds for every $j, 1 \leq j \leq l$. Multiplying by $t_{j}$ and summing up from $j=1$ to $l$, we find

$$
\int_{\Omega} a|\nabla u|^{2} \cosh (u)=\sum_{j=1}^{l} \lambda_{j} t_{j} \int_{\partial \Omega} a v_{j} \sinh u .
$$

On the finite dimensional space $V^{-}$the functional (2.6) takes the form

$$
\begin{gathered}
E_{\lambda}(u) \equiv f\left(t_{1}, \ldots, t_{l}\right)=\sum_{i, j=1}^{l} \frac{t_{i} t_{j}}{2} \int_{\Omega} a \nabla v_{i} \cdot \nabla v_{j}-\lambda \int_{\partial \Omega} a(\cosh u-1) \\
=\frac{1}{2} \sum_{i=1}^{l} t_{i}^{2}-\lambda \int_{\partial \Omega} a(\cosh u-1),
\end{gathered}
$$

where we used orthogonality and normalization of $v_{i}$ with respect to the inner product defined by the equivalent norm (2.4). Then, we have

$$
\begin{aligned}
& \left(\sum_{j=1}^{l} \lambda_{j} t_{j} \partial_{t_{j}}\right) f\left(t_{1}, \ldots, t_{l}\right)=\sum_{j=1}^{l} \lambda_{j} t_{j}^{2}-\lambda \sum_{j=1}^{l} \lambda_{j} t_{j} \int_{\partial \Omega} a v_{j} \sinh u \\
& \leq \lambda_{l} \sum_{j=1}^{l} t_{j}^{2}-\lambda \int_{\Omega} a|\nabla u|^{2} \cosh u=\int_{\Omega} a|\nabla u|^{2}\left[\lambda_{l}-\lambda \cosh u\right] .
\end{aligned}
$$

Now, it can be proved (see [7, Lemma 3.5]) that the last term is strictly negative for $\|u\|_{a}=\sqrt{t_{1}^{2}+\cdots t_{l}^{2}}$ large enough. But the first term is the derivative of the function $f$ along the curves

$$
t_{1}=c_{1} e^{\lambda_{1} s}, \ldots, t_{l}=c_{l} e^{\lambda_{l} s}, \quad s \in \mathbb{R}
$$

(orthogonal to the hypersurfaces $\lambda_{1} t_{1}^{2}+\cdots+\lambda_{l} t_{l}^{2}=$ constant); hence, for large $\sqrt{t_{1}^{2}+\cdots t_{l}^{2}}$, the function $f$ is strictly decreasing along these curves.

We conclude that $f(u)<0$ for $u \in V^{-}$with $\|u\|_{a}$ large enough; since $f$ is continuous and $V^{-}$has finite dimension, we have

$$
\sup _{u \in V^{-}} f(u)=c_{\infty}<\infty .
$$

We are left to show that we may allow $\lambda_{n_{1}}=0$ in (2.14). This can be proved by the same arguments as in the proof of Lemma 3.5 of [8].

2.2. Proof of Theorem 1.1. To prove existence and even multiplicity of solutions to problem (1.7) for every $\lambda>0$, we will apply the symmetric mountain pass lemma (see [11, Theorem 6.3]); thus, we need to show that the functional (2.6) satisfies the Palais-Smale condition; to this aim the following estimate plays a key role:

$$
4(\cosh u-1) \leq u \sinh u+u^{2} .
$$


Proposition 2.5. Let $z_{m} \in H^{1}(\Omega)$ be a sequence such that $E_{\lambda}\left(z_{m}\right) \rightarrow c$ and $E_{\lambda}^{\prime}\left(z_{m}\right) \rightarrow 0$ in $H^{1}(\Omega)^{\prime}$. Then, the sequence $z_{m}$ is bounded and the functional (2.6) satisfies the Palais-Smale condition.

Proof. Assume by contradiction (considering a subsequence if necessary) that $\left\|z_{m}\right\|_{a} \rightarrow+\infty$, and define $t_{m}=\left\|z_{m}\right\|_{a}, u_{m}=t_{m}^{-1} z_{m}$. Substituting in the condition $E_{\lambda}^{\prime}\left(z_{m}\right) u=o(1)\|u\|_{a}$, we get

$$
\int_{\Omega} a \nabla u_{m} \nabla u-\lambda \int_{\partial \Omega} a \frac{\sinh \left(t_{m} u_{m}\right)}{t_{m}} u=o(1)\|u\|_{a} / t_{m} .
$$

Since $u_{m}$ is bounded in $H^{1}(\Omega)$, there is a subsequence (still denoted by $u_{m}$ ) such that $u_{m}$ converges weakly in $H^{1}(\Omega)$ and $\left.u_{m}\right|_{\partial \Omega}$ converges strongly in $L^{2}(\partial \Omega)$; it can be proved (see [7, Proposition 4.2]) that $\left.u_{m}\right|_{\partial \Omega} \rightarrow 0$ a.e.

By choosing $u=u_{m}$ in (2.21) we get

$$
\int_{\Omega} a\left|\nabla u_{m}\right|^{2}-\lambda \int_{\partial \Omega} a \frac{\sinh \left(t_{m} u_{m}\right)}{t_{m}} u_{m}=o\left(1 / t_{m}\right) .
$$

On the other hand, from $E_{\lambda}\left(z_{m}\right) \rightarrow c$ we also get

$$
\frac{1}{2} \int_{\Omega} a\left|\nabla u_{m}\right|^{2}-\lambda \int_{\partial \Omega} a \frac{\cosh \left(t_{m} u_{m}\right)-1}{t_{m}^{2}}=O\left(1 / t_{m}^{2}\right) .
$$

By comparison of (2.22) and (2.23) and by taking (2.20) into account we find

$$
\begin{aligned}
0 & =\lambda \int_{\partial \Omega} a\left[2 \frac{\cosh \left(t_{m} u_{m}\right)-1}{t_{m}^{2}}-\frac{\sinh \left(t_{m} u_{m}\right)}{t_{m}} u_{m}\right]+o\left(1 / t_{m}\right) \\
& \leq-2 \lambda \int_{\partial \Omega} a \frac{\cosh \left(t_{m} u_{m}\right)-1}{t_{m}^{2}}+\lambda \int_{\partial \Omega} a u_{m}^{2}+o\left(1 / t_{m}\right) .
\end{aligned}
$$

By recalling that $u_{m} \rightarrow 0$ in $L^{2}(\partial \Omega)$ we finally get

$$
0 \leq-2 \lambda \int_{\partial \Omega} a \frac{\cosh \left(t_{m} u_{m}\right)-1}{t_{m}^{2}}+o(1) .
$$

By the above relation and again by (2.23) we conclude

$$
\int_{\Omega} a\left|\nabla u_{m}\right|^{2} \rightarrow 0
$$

so that $\left\|u_{m}\right\|_{a} \rightarrow 0$, thus contradicting $\left\|u_{m}\right\|_{a}=1$. Then, the norm sequence $\left\|z_{m}\right\|_{a}$ is bounded and the same holds for $\left\|z_{m}\right\|$. We can write

$$
z_{m}=c_{m}+\tilde{z}_{m},
$$

where $c_{m}$ is a bounded sequence and $\tilde{z}_{m}$ is bounded in $H_{a}^{1}(\Omega)$ (see definition (2.2)).

Now, the linear map $L: H_{a}^{1}(\Omega) \rightarrow H^{1}(\Omega)^{\prime}$

$$
L(u) \varphi=\int_{\Omega} a \nabla u \nabla \varphi
$$

is boundedly invertible (by the Lax-Milgram theorem) while the operator

$$
T(u) \varphi=\int_{\partial \Omega} a \sinh u \varphi
$$

Copyright $@$ by SIAM. Unauthorized reproduction of this article is prohibited. 
maps bounded sets in $H^{1}(\Omega)$ to relatively compact sets in $H^{1}(\Omega)^{\prime}$ (the result follows by an obvious extension of the arguments in [6, Lemmas 2.1 and 2.2]). By standard results [11, Proposition 2.2], it follows that $\tilde{z}_{m}$ is relatively compact in $H_{a}^{1}(\Omega)$; then, by the above decomposition, the same holds for $z_{m}$ in $H^{1}(\Omega)$.

Proof of Theorem 1.1. By Lemmas 2.3 and 2.4, for any positive integer $m$ there exist two closed subspaces $V^{+}, V^{-}$of $H^{1}(\Omega)$ with $\operatorname{dim} V^{-}-\operatorname{codim} V^{+}=m$, and positive constants $R, c_{0}, c_{\infty}$ (the last one depending on $m$ ) such that
(a) $\quad E_{\lambda}(u) \geq c_{0} \quad \forall u \in V^{+}, \quad\|u\|_{a}=R$
(b) $E_{\lambda}(u) \leq c_{\infty} \quad \forall u \in V^{-}$.

Then, by Theorem 2.4 of [1], the functional $E_{\lambda}$ possesses at least $m$ distinct pairs of critical points, corresponding to critical levels $c_{k}(\lambda), k=1,2, \ldots, m$, given by

$$
c_{k}(\lambda)=\inf _{A \in \Sigma_{k}} \sup _{u \in A} E_{\lambda}(u)
$$

where $\Sigma_{k}$ is the set of closed, symmetric sets $A \subset H^{1}(\Omega)$ such that $\gamma^{*}(A) \geq k$ and $\gamma^{*}$ is the pseudoindex related to the Krasnoselski genus $\gamma$ and to the subset $S_{R} \cap V^{+}$ (see [1, Definition 2.8]).

Moreover,

$$
c_{0} \leq c_{1} \leq c_{2} \leq \cdots \leq c_{m} \leq c_{\infty} .
$$

Since this conclusion holds for arbitrary $m$, we get infinitely many critical points; hence, problem (1.7) has infinitely many solutions in $H^{1}(\Omega)$. By standard regularity results [3], if $\Omega$ is smooth and $a \in \mathcal{C}^{\infty}(\bar{\Omega})$, we have $u \in \mathcal{C}^{\infty}(\bar{\Omega})$.

Remark 2.6. In the degenerate case $c_{k}=\cdots=c_{k+r}=c$ (with $k \geq 1$ and $k+r \leq m)$ it was shown in [1] that $\gamma\left(K_{c}\right) \geq r+1 \geq 2$, where $K_{c}$ is the set of critical points at level $c$; since a finite set (not containing the origin) has genus 1 , it follows that $E_{\lambda}$ has infinitely many critical points at level $c$.

Remark 2.7. The results of existence and multiplicity obtained in [6] correspond to the case $a=1$ of Theorem 1.1 above.

From the discussion given in the introduction, the existence of solutions of problem (1.4) stated in Corollary 1.2 easily follows.

2.3. Estimates and limits for the variational solutions. As discussed in the introduction, we now prove lower and upper bounds for the variational solutions obtained in the previous subsection; more precisely, we show that a branch of solutions corresponding to any of the critical levels $c_{k}(\lambda)$ blows up in energy (as well as in the Dirichlet seminorm) at the rate $\log (1 / \lambda)$ for $\lambda \rightarrow 0^{+}$, while the corresponding normal currents stay bounded in $L^{1}(\partial \Omega)$. In what follows, we shall assume that $\Omega$ is bounded and of class $\mathcal{C}^{2, \alpha}$, which implies that we are dealing with classical solutions $\left(\mathcal{C}^{\infty}(\Omega) \cap \mathcal{C}^{1, \alpha}(\bar{\Omega})\right)$.

We first establish the lower bounds; by observing that a solution $u$ to (1.7) satisfies

$$
0=\int_{\partial \Omega} a \partial_{\nu} u=\lambda \int_{\partial \Omega} a \sinh u
$$

we may write $u=u^{0}+s, s \in \mathbb{R}$, where $u^{0}$ solves the problem

$$
\left\{\begin{array}{l}
\Delta_{a} u^{0}=0 \quad \text { in } \Omega \\
\partial_{\nu} u^{0}=\lambda \sinh \left(u^{0}+s\right) \quad \text { on } \partial \Omega
\end{array}\right.
$$

Copyright $@$ by SIAM. Unauthorized reproduction of this article is prohibited. 
and

$$
\int_{\partial \Omega} a u^{0}=0, \quad \int_{\partial \Omega} a \sinh \left(u^{0}+s\right)=0 .
$$

By elementary calculations, the second identity yields

$$
s=s\left(u^{0}\right) \equiv \frac{1}{2} \log \frac{\int_{\partial \Omega} a e^{-u^{0}}}{\int_{\partial \Omega} a e^{u^{0}}} .
$$

Now, following $[6]$ and by recalling the bound (1.8), one first proves the inequality

$$
\left|s\left(u^{0}\right)\right| \leq C_{1}+C_{2}\left\|\nabla u^{0}\right\|^{2}
$$

(with positive constants $C_{1}, C_{2}$ depending only on $\Omega$ and $a$ ) and subsequently the following lower estimates for the solutions to (1.7):

$$
E_{\lambda}(u) \geq A \log \left(\frac{1}{\lambda}\right)-B, \quad\|\nabla u\|^{2} \geq A \log \left(\frac{1}{\lambda}\right)-B,
$$

where $E_{\lambda}$ is the energy functional defined in (2.6) and the positive constants $A, B$ are independent of $\lambda$ and $u$.

By considering the variational solutions given by Theorem 1.1, we now provide upper estimates of the critical values $c_{k}(\lambda)(2.25)$. We first remark that any finite dimensional subspace $V$ with $\operatorname{dim} V>\operatorname{codim} V^{+}\left(V^{+}\right.$being the subspace defined in (2.12)) satisfies $V \in \Sigma_{k}$ for $k \leq \operatorname{dim} V-\operatorname{codim} V^{+}$(this is related to the socalled intersection lemma; see [11, Lemma 6.4] and the proof of Theorem 2.4 of [1]). Moreover, since for $\lambda \rightarrow 0^{+}$we can take $V^{+}=H_{a}^{1}$, which is the subspace of codimension 1 defined in (2.2), we may assume $\operatorname{dim} V=k+1$.

Now, let us choose $k+1$ distinct points on the boundary $\partial \Omega$ and, for $\epsilon>0$, define

$$
V_{\epsilon} \equiv \operatorname{span}\left\{-\log \left(\epsilon^{2}+\left|x-\sigma_{j}\right|^{2}\right), \quad j=1,2, \ldots, k+1\right\} .
$$

By the estimates of Lemmas 3.4 and 3.5 in [6] (with obvious modifications due to the expression (2.6) of $E_{\lambda}$ ) one finds, for $\lambda$ sufficiently small and by taking $\epsilon$ of order $\lambda$,

$$
\max _{u \in V_{\epsilon}} E_{\lambda}(u) \leq C_{*} \log \left(\frac{1}{\lambda}\right)
$$

for some constant $C_{*}$ depending only on $k, a$, and $\Omega$. Since by the previous remark we have $V_{\epsilon} \in \Sigma_{k}$, the bound

$$
c_{k}(\lambda) \leq C_{*} \log \left(\frac{1}{\lambda}\right)
$$

follows.

Finally, the estimate (2.29) together with an elementary lemma in integration theory (Lemma 3.6 of [6]) yield the above-mentioned bound on the normal current.

Proposition 2.8. Let $\lambda>0$ be small enough so that (2.29) holds. For $k \geq 1$, let $u_{k, \lambda}$ be the variational solutions obtained in Theorem 1.1 with $E_{\lambda}\left(u_{k}\right)=c_{k}(\lambda)$. Then, there exists a constant $D_{*}$ depending only on $k, a$, and $\Omega$ such that

$$
\int_{\partial \Omega}\left|\frac{\partial u_{k, \lambda}}{\partial \nu}\right|=\lambda \int_{\partial \Omega}\left|\sinh \left(u_{k, \lambda}\right)\right| \leq D_{*} .
$$

Copyright $@$ by SIAM. Unauthorized reproduction of this article is prohibited. 
The proof follows by a trivial modification of the proof of Corollary 3.7 in [6].

By exploiting the previous estimates it is possible to describe the behavior of a sequence of solutions $u_{\lambda_{n}}=u_{k, \lambda_{n}}$, where $k \geq 1$ is fixed and $\lambda_{n} \rightarrow 0$. The results, which will be stated below, can be proved by an adaptation of the arguments of section 4 of [6]; the only nontrivial change is the use of a representation formula for a classical solution $w$ of the Neumann problem

$$
\Delta_{a} w=0 \quad \text { in } \Omega, \quad \frac{\partial w}{\partial \nu}=f \quad \text { on } \partial \Omega, \quad \int_{\partial \Omega} a w=0
$$

(where $f$ is such that $\int_{\partial \Omega} a f=0$ ), which extends the usual layer potential representation for harmonic functions. We prove here this formula.

Lemma 2.9. Let $w$ be the solution to (2.31). Then

$$
w(y)=\frac{1}{2 \pi a(y)} \int_{\partial \Omega} a(\sigma) G_{a}(\sigma, y) f(\sigma) d \sigma,
$$

where $G_{a}$ is the Green's function defined in (1.10). ${ }^{1}$

Proof. We first remark that, by the boundary condition in (1.10) and by the normalization condition for $w$ in (2.31) above, the following holds:

$$
\int_{\partial \Omega} a(\sigma) w(\sigma) \partial_{\nu} G_{a}(\sigma, y) d \sigma=2 \pi a(y) w(y) .
$$

Then, we can compute

$$
\begin{gathered}
\int_{\partial \Omega} a(\sigma) G_{a}(\sigma, y) f(\sigma) d \sigma=\int_{\partial \Omega} a(\sigma) G_{a}(\sigma, y) \partial_{\nu} w(\sigma) d \sigma \\
-\int_{\partial \Omega} a(\sigma) w(\sigma) \partial_{\nu} G_{a}(\sigma, y) d \sigma+2 \pi a(y) w(y) \\
=\int_{\Omega} \operatorname{div}\left[G_{a}(x, y) a(x) \nabla_{x} w(x)-w(x) a(x) \nabla_{x} G_{a}(x, y)\right] d x+2 \pi a(y) w(y) \\
=\int_{\Omega}\left[G_{a}(x, y) \operatorname{div}\left(a(x) \nabla_{x} w(x)\right)-w(x) \operatorname{div}\left(a(x) \nabla_{x} G_{a}(x, y)\right)\right] d x+2 \pi a(y) w(y) \\
=2 \pi a(y) w(y) .
\end{gathered}
$$

Hence, formula (2.32) follows.

As discussed above, one can now reproduce all the estimates proved in [6] (in particular, those in Lemma 4.2 of [6]), which leads to the following result.

Proposition 2.10. Let $u_{\lambda_{n}} \in H^{1}(\Omega), \lambda_{n} \rightarrow 0^{+}$, be a sequence of solutions to (1.7) given by Theorem 1.1. Then, there exists a subsequence, also denoted by $u_{\lambda_{n}}$, a regular finite measure $m$ on $\partial \Omega$, and a finite set of points $\left\{x^{(i)}\right\}_{i=1}^{N} \subset \partial \Omega, N \geq 1$, such that

$$
\lambda_{n}\left|\sinh \left(u_{\lambda_{n}}\right)\right| \rightarrow m
$$

on $\partial \Omega$ in the sense of measures and the points $x^{(i)}, i=1, \ldots, N$, are exactly the points at which $m$ has point masses. The same points also represent the blow up points for the sequence

$$
u_{\lambda_{n}}^{0}=u_{\lambda_{n}}-\frac{1}{\int_{\partial \Omega} a} \int_{\partial \Omega} a u_{\lambda_{n}}
$$

\footnotetext{
${ }^{1} \mathrm{By}$ an abuse of notation we write here $a(\sigma)$ (and similarly $G_{a}(\sigma, y)$, etc.) instead of $\left.a(x)\right|_{\partial \Omega}$.
}

Copyright (c) by SIAM. Unauthorized reproduction of this article is prohibited. 
in the sense that

$$
\left\{x^{(i)}\right\}_{i=1}^{N}=\left\{x \in \bar{\Omega}: \exists x_{n} \rightarrow x, x_{n} \in \bar{\Omega}, \text { with }\left|u_{\lambda_{n}}^{0}\left(x_{n}\right)\right| \rightarrow \infty\right\} .
$$

3. Blowing up solutions. We now show that there are solutions to problem (1.7) which concentrate at isolated critical points $\xi_{1}$ and $\xi_{2}$, say, of $a$ constrained on $\partial \Omega$ as $\lambda \rightarrow 0$. By the previous discussion, this corresponds to building up solutions to problem (1.4) that concentrate positively and negatively along two geodesics of the boundary of $\mathcal{D}$ which are nothing but the $\mathcal{G}$-orbits of $\xi_{1}$ and $\xi_{2}$, respectively.

This section is organized as follows. In subsection 3.1, we write an approximate solution for problem (1.7). In subsection 3.2 we study an associated linear problem, and in subsection 3.3 we reduce our nonlinear problem to a finite dimensional one. In subsection 3.4 we study the reduced problem and we prove Theorem 1.3.

3.1. The approximate solution. To define an approximate solution for the problem (1.7), a key ingredient is given by the solutions of the following problem:

$$
\left\{\begin{array}{l}
\Delta v=0 \quad \text { in } \mathbb{R}_{+}^{2}, \\
\frac{\partial v}{\partial \nu}=e^{v} \quad \text { on } \partial \mathbb{R}_{+}^{2},
\end{array}\right.
$$

where $\mathbb{R}_{+}^{2}$ denotes the upper half plane $\left\{\left(x_{1}, x_{2}\right): x_{2}>0\right\}$ and $\nu$ is the unit exterior normal to $\partial \mathbb{R}_{+}^{2}$.

The solutions of (3.1) are given by

$$
w_{t, \mu}\left(x_{1}, x_{2}\right)=\log \frac{2 \mu}{\left(x_{1}-t\right)^{2}+\left(x_{2}+\mu\right)^{2}},
$$

where $t \in \mathbb{R}$ and $\mu>0$ are parameters.

Let us provide an approximation for the solution of our problem. Let

$$
u_{j}^{\lambda}(x)=\log \frac{2 \mu_{j}}{\left|x-\xi_{j}-\lambda \mu_{j} \nu_{j}\right|^{2}}, \quad j=1,2, \quad \xi_{j} \in \partial \Omega, \mu_{j}>0 .
$$

In order to satisfy the equation $\Delta_{a} u=0$, we need an additional term $H_{j}^{\lambda}$ defined as follows: let $H_{j}^{\lambda}(x)$ be the unique solution of

$$
\left\{\begin{array}{l}
-\Delta_{a} H_{j}^{\lambda}=\nabla \log a \cdot \nabla u_{j}^{\lambda} \quad \text { in } \Omega \\
\frac{\partial H_{j}^{\lambda}}{\partial \nu}=-\frac{\partial u_{j}^{\lambda}}{\partial \nu}+\lambda e^{u_{j}^{\lambda}}-\lambda \frac{1}{\int_{\partial \Omega} a} \int_{\partial \Omega} a e^{u_{j}^{\lambda}} \quad \text { on } \partial \Omega \\
\int_{\partial \Omega} H_{j}^{\lambda} d x=-\int_{\partial \Omega} u_{j}^{\lambda} d x
\end{array}\right.
$$

Now we set

$$
U_{\lambda}(x)=\left[u_{1}^{\lambda}(x)+H_{1}^{\lambda}(x)\right]-\left[u_{2}^{\lambda}(x)+H_{2}^{\lambda}(x)\right]
$$

and look for a solution of (1.7) in the form

$$
u_{\lambda}(x)=U_{\lambda}(x)+\Phi_{\lambda}(x)
$$

Copyright (c) by SIAM. Unauthorized reproduction of this article is prohibited. 
The lower-order term $\Phi_{\lambda}$ will satisfy some suitable orthogonality conditions (see (3.12)).

Let $G_{a}(x, y)$ be defined as in (1.10), and let $H_{a}(x, y)$ be the regular part of $G_{a}(x, y)$ defined as in (1.11). In the following we will write simply $G(x, y)$ instead of $G_{1}(x, y)$ and $H(x, y)$ instead of $H_{1}(x, y)$.

It is immediate to see that $H_{a}(x, y)$ solves the following problem:

$$
\left\{\begin{array}{l}
\left(\Delta_{a}\right)_{x} H_{a}(x, y)=2 \nabla \log a \cdot \frac{x-y}{|x-y|^{2}} \quad \text { in } \Omega, \\
\frac{\partial H_{a}}{\partial \nu_{x}}(x, y)=-\frac{2 \pi}{\int_{\partial \Omega} a(x)} a(y)+\frac{2(x-y) \cdot \nu(x)}{|x-y|^{2}} \quad \text { on } \partial \Omega .
\end{array}\right.
$$

The function $H_{j}^{\lambda}$ can be estimated in terms of $H_{a}(x, y)$. Indeed, the following result holds.

Lemma 3.1. For any $\alpha \in(0,1)$,

$$
H_{j}^{\lambda}(x)=H_{a}\left(x, \xi_{j}\right)-\log 2 \mu_{j}+O\left(\lambda^{\alpha}\right)
$$

uniformly in $\bar{\Omega}$.

Proof. The boundary condition satisfied by $H_{j}^{\lambda}$ is

$$
\begin{aligned}
\frac{\partial H_{j}^{\lambda}}{\partial \nu} & =-\frac{\partial u_{j}^{\lambda}}{\partial \nu}+\lambda e^{u_{j}^{\lambda}}-\frac{\lambda}{\int_{\partial \Omega} a} \int_{\partial \Omega} a e^{u_{j}^{\lambda}} \\
& =2 \lambda \mu_{j} \frac{1-\nu\left(\xi_{j}\right) \cdot \nu(x)}{\left|x-\xi_{j}-\lambda \mu_{j} \nu\left(\xi_{j}\right)\right|^{2}}+2 \frac{\left(x-\xi_{j}\right) \cdot \nu(x)}{\left|x-\xi_{j}-\lambda \mu_{j} \nu\left(\xi_{j}\right)\right|^{2}}-\frac{\lambda}{\int_{\partial \Omega} a} \int_{\partial \Omega} a e^{u_{j}^{\lambda}}
\end{aligned}
$$

As $\lambda \rightarrow 0$ we get

$$
\begin{aligned}
\lambda \int_{\partial \Omega} a e^{u_{j}^{\lambda}} & =\lambda \int_{\partial \Omega} a(x) \frac{2 \mu_{j}}{\left|x-\xi_{j}-\lambda \mu_{j} \nu\left(\xi_{j}\right)\right|^{2}}=2 \int_{\frac{\partial \Omega-\xi_{j}}{\lambda \mu_{j}}} \frac{a\left(\xi_{j}+\lambda \mu_{j} y\right)}{|y-\nu(0)|^{2}} \\
& =2 a\left(\xi_{j}\right) \int_{\frac{\partial \Omega-\xi_{j}}{\lambda \mu_{j}}} \frac{1}{|y-\nu(0)|^{2}}+O\left(\lambda\left|\nabla a\left(\xi_{j}\right)\right| \mu_{j} \int_{\frac{\partial \Omega-\xi_{j}}{\lambda \mu_{j}}} \frac{1}{|y-\nu(0)|^{2}}\right) \\
& =2 a\left(\xi_{j}\right)\left(\int_{-\infty}^{+\infty} \frac{1}{1+t^{2}} d t-O\left(\int_{\lambda^{-1} \mu_{j}^{-1}} \frac{1}{1+t^{2}} d t\right)\right) \\
& =a\left(\xi_{j}\right)\left(2 \pi+O\left(\arctan \left(\lambda \mu_{j}\right)^{-1}-\frac{\pi}{2}\right)\right)+O\left(\lambda \mu_{j}\right) \\
& =2 \pi a\left(\xi_{j}\right)+O\left(\arctan \left(\lambda \mu_{j}\right)\right)+O\left(\lambda \mu_{j}\right) \\
& =2 \pi a\left(\xi_{j}\right)+O\left(\lambda \mu_{j}\right) .
\end{aligned}
$$

Let us consider the difference

$$
z_{\lambda}^{\xi_{j}}(x)=H_{j}^{\lambda}(x)-H_{a}\left(x, \xi_{j}\right)+\log 2 \mu_{j} .
$$

Hence $z_{\lambda}$ solves the following problem:

$$
\left\{\begin{array}{l}
-\Delta_{a} z_{\lambda}^{\xi_{j}}=-\Delta_{a} H_{j}^{\lambda}+\Delta_{a} H_{a}\left(x, \xi_{j}\right) \\
\frac{\partial z_{\lambda}^{\xi_{j}}}{\partial \nu}=\frac{\partial H_{j}^{\lambda}}{\partial \nu}-\frac{\partial H_{a}}{\partial \nu}
\end{array}\right.
$$

Copyright $@$ by SIAM. Unauthorized reproduction of this article is prohibited. 
namely

$$
\left\{\begin{array}{l}
-\Delta_{a} z_{\lambda}^{\xi_{j}}=\nabla \log a(x) \nabla\left[\log \frac{2 \mu_{j}}{\left|x-\xi_{j}-\lambda \mu_{j} \nu_{j}\right|^{2}}-\log \frac{1}{\left|x-\xi_{j}\right|^{2}}\right] \\
\frac{\partial z_{\lambda}^{\xi_{j}}}{\partial \nu}=O\left(\lambda \mu_{j}\right) .
\end{array}\right.
$$

As in Lemma 3.1 of [4], it follows that for any $p>1$,

$$
\left\|\frac{\partial z_{\lambda}^{\xi_{j}}}{\partial \nu}\right\|_{L^{p}(\partial \Omega)} \leq c \lambda^{\frac{1}{p}}
$$

Moreover, again as in Lemma 3.1 of [4], we get

$$
\begin{aligned}
\| \log \frac{1}{\left|x-\xi_{j}\right|^{2}} & -\log \frac{1}{\left|x-\xi_{j}-\lambda \mu_{j} \nu\left(\xi_{j}\right)\right|^{2}} \|_{L^{p}(\Omega)}^{p} \\
& =\int_{B_{\lambda \mu_{j}}\left(\xi_{j}\right) \cap \Omega} \cdots+\int_{\Omega \backslash B_{\lambda \mu_{j}}\left(\xi_{j}\right)} \cdots=I_{1}+I_{2} .
\end{aligned}
$$

Now

$$
\left|I_{1}\right| \leq C \lambda^{2}\left(\log \frac{1}{\lambda}\right)^{p}
$$

while for $p \in(1,2)$

$$
\left|I_{2}\right| \leq C \lambda^{p}
$$

In conclusion, for any $p \in(1,2)$

$$
\left\|\log \frac{1}{\left|x-\xi_{j}\right|^{2}}-\log \frac{1}{\left|x-\xi_{j}-\lambda \mu_{j} \nu\left(\xi_{j}\right)\right|^{2}}\right\|_{L^{p}(\Omega)} \leq c \lambda .
$$

By $L^{p}$-theory,

$$
\left\|z_{\lambda}^{\xi_{j}}\right\|_{W^{1+s, p}(\Omega)} \leq C\left(\left\|\frac{\partial z_{\lambda}^{\xi_{j}}}{\partial \nu}\right\|_{L^{p}(\partial \Omega)}+\left\|\Delta_{a} z_{\lambda}^{\xi_{j}}\right\|_{L^{p}(\Omega)}\right) \leq C \lambda^{\frac{1}{p}}
$$

for any $0<s<\frac{1}{p}$. By Morrey's embedding we obtain

$$
\left\|z_{\lambda}^{\xi_{j}}\right\|_{C^{\gamma}(\bar{\Omega})} \leq c \lambda^{\frac{1}{p}}
$$

for any $0<\gamma<\frac{1}{2}+\frac{1}{p}$. This proves the result with $\alpha=\frac{1}{p}$.

Moreover, the function $H_{a}(x, y)$ can be expanded in terms of $H(x, y)$. The following expansion is proved in Lemma 2.1 of [13].

Lemma 3.2. Let $H_{a, y}(x)=H_{a}(x, y)$ for any $y \in \Omega$. Then $y \rightarrow H_{a, y}$ is a continuous map from $\Omega$ into $C^{0, \gamma}(\bar{\Omega})$ for any $\gamma \in(0,1)$. It follows that

$$
H_{a}(x, y)=H(x, y)+\nabla \log a(y) \cdot \nabla\left(|x-y|^{2} \log |x-y|\right)+\mathcal{H}(x, y),
$$

Copyright $@$ ( ) by SIAM. Unauthorized reproduction of this article is prohibited. 
where $y \rightarrow \mathcal{H}(\cdot, y)$ is a continuous map from $\Omega$ into $C^{1, \gamma}(\bar{\Omega})$ for all $\gamma \in(0,1)$. Furthermore, the function $(x, y) \rightarrow \mathcal{H}(x, y) \in C^{1}(\Omega \times \Omega)$; in particular, $x \rightarrow H_{a}(x, x) \in$ $C^{1}(\Omega)$.

We consider now the following change of variables:

$$
x=\lambda y, \quad y \in \Omega_{\lambda} \equiv \frac{\Omega}{\lambda}, \quad v(y)=u(\lambda y) .
$$

Then $u$ is a solution to problem (1.7) if and only if $v$ solves the problem

$$
\begin{cases}\Delta_{a_{\lambda}} v=0 & \text { in } \Omega_{\lambda}, \\ \frac{\partial v}{\partial \nu}=2 \lambda^{2} \sinh v & \text { on } \partial \Omega_{\lambda},\end{cases}
$$

where $a_{\lambda}(y):=a(\lambda y)$. In the expanded domain $\Omega_{\lambda}, U_{\lambda}(x)$ becomes

$$
V(y)=\sum_{j=1}^{2}(-1)^{j-1}[\underbrace{\log \frac{2 \mu_{j}}{\left|y-\xi_{j}^{\prime}-\mu_{j} \nu_{j}^{\prime}\right|^{2}}-2 \log \lambda}_{=u_{j}^{\lambda}(\lambda y)}+H_{j}^{\lambda}(\lambda y)],
$$

where $\xi_{j}^{\prime}=\lambda^{-1} \xi_{j}$ and $\nu_{j}^{\prime}=\nu\left(\xi_{j}^{\prime}\right)$. Therefore,

$$
v(y)=V(y)+\phi(y), \quad y \in \Omega_{\lambda},
$$

will be a solution of (3.2) provided $\phi$ solves

$$
\left\{\begin{array}{l}
\Delta_{a_{\lambda}} \phi=0 \quad \text { in } \Omega_{\lambda}, \\
\frac{\partial \phi}{\partial \nu}-\mathcal{W} \phi=\mathcal{R}+\mathcal{N}(\phi) \quad \text { on } \partial \Omega_{\lambda},
\end{array}\right.
$$

where we set

$$
\begin{gathered}
\mathcal{W}(y):=2 \lambda^{2} \cosh V(y), \\
\mathcal{R}(y):=-\left[\frac{\partial V}{\partial \nu}-2 \lambda^{2} \sinh V\right](y),
\end{gathered}
$$

and

$$
\mathcal{N}(\phi)=2 \lambda^{2}[\sinh (V+\phi)-\sinh V-(\cosh V) \phi] .
$$

First, we prove that $V$ is a good approximation for a solution to (3.2), provided the parameters $\mu_{1}$ and $\mu_{2}$ are suitably choosen.

Lemma 3.3. Assume

$$
\log 2 \mu_{1}=H_{a}\left(\xi_{1}, \xi_{1}\right)-G_{a}\left(\xi_{1}, \xi_{2}\right) \quad \text { and } \quad \log 2 \mu_{2}=H_{a}\left(\xi_{2}, \xi_{2}\right)-G_{a}\left(\xi_{2}, \xi_{1}\right) .
$$

Then, for any $\alpha \in(0,1)$, there exists a positive constant $C$ independent of $\lambda$ such that, for any $y \in \Omega_{\lambda}$,

$$
|\mathcal{R}(y)| \leq \lambda^{\alpha} \sum_{j=1}^{2} \frac{1}{1+\left|y-\xi_{j}^{\prime}\right|} \quad \forall y \in \Omega_{\lambda},
$$

Copyright $@$ by SIAM. Unauthorized reproduction of this article is prohibited. 
and

$$
\mathcal{W}(y)=\sum_{j=1}^{2} \frac{2 \mu_{j}}{\left|y-\xi_{j}^{\prime}-\mu_{j} \nu_{j}^{\prime}\right|^{2}}\left(1+\theta_{\lambda}(y)\right), \quad \text { with } \quad\left|\theta_{\lambda}(y)\right| \leq C \lambda^{\alpha}+C \lambda \sum_{j=1}^{2}\left|y-\xi_{j}^{\prime}\right| .
$$

Proof. By Lemma 3.1 we deduce that if $\left|y-\xi_{j}^{\prime}\right| \leq \frac{\delta}{\lambda}$,

$$
\begin{aligned}
& H_{1}^{\lambda}(\lambda y)-\left(\log \frac{2 \mu_{2}}{\lambda^{2}\left|y-\xi_{2}^{\prime}-\mu_{2} \nu\left(\xi_{2}^{\prime}\right)\right|^{2}}+H_{2}^{\lambda}(\lambda y)\right) \quad\left(\text { setting } z:=y-\xi_{1}^{\prime}\right) \\
& =\left(H_{a}\left(\lambda z+\xi_{1}, \xi_{1}\right)-\log 2 \mu_{1}\right) \\
& -\left(\log 2 \mu_{2}+\log \frac{1}{\left|\lambda z+\left(\xi_{1}-\xi_{2}\right)-\lambda \mu_{2} \nu\left(\xi_{2}^{\prime}\right)\right|^{2}}+H_{a}\left(\lambda z+\xi_{1}, \xi_{2}\right)-\log 2 \mu_{2}\right)+O\left(\lambda^{\alpha}\right) \\
& =\underbrace{H_{a}\left(\xi_{1}, \xi_{1}\right)-\log 2 \mu_{1}-G_{a}\left(\xi_{1}, \xi_{2}\right)}_{=0 \text { because of }(3.7)}+O\left(\lambda^{\alpha}\right)+O(\lambda|z|) \\
& =O\left(\lambda^{\alpha}\right)+O\left(\lambda\left|y-\xi_{1}^{\prime}\right|\right)
\end{aligned}
$$

and, in a similar way,

$$
H_{2}^{\lambda}(\lambda y)-\left(\log \frac{2 \mu_{1}}{\lambda^{2}\left|y-\xi_{1}^{\prime}-\mu_{1} \nu\left(\xi_{1}^{\prime}\right)\right|^{2}}+H_{1}^{\lambda}(\lambda y)\right)=O\left(\lambda^{\alpha}\right)+O\left(\lambda\left|y-\xi_{2}^{\prime}\right|\right) .
$$

Therefore, the proof follows exactly as in Lemma 3 of [5].

3.2. A linear problem. The key ingredient in this section is the linearization of problem (3.1) around the solution $w_{0, \mu}$, namely the problem

$$
\begin{cases}\Delta \phi=0 & \text { in } \mathbb{R}_{+}^{2}, \\ \frac{\partial \phi}{\partial \nu}=\frac{2 \mu}{x_{1}^{2}+\mu^{2}} \phi & \text { on } \partial \mathbb{R}_{+}^{2} .\end{cases}
$$

In [5] it has been proved that the following result holds.

LEMMA 3.4. Any bounded solution of (3.10) is a linear combination of the functions

$$
z_{0}(x)=1-2 \mu \frac{x_{2}+\mu}{x_{1}^{2}+\left(x_{2}+\mu\right)^{2}}
$$

and

$$
z_{1}(x)=-2 \frac{x_{1}}{x_{1}^{2}+\left(x_{2}+\mu\right)^{2}}
$$

Now, let us assume that the points $\xi_{1}, \xi_{2} \in \partial \Omega$ are uniformly separated, namely $\left|\xi_{1}-\xi_{2}\right| \geq d$ for some $d>0$ which does not depend on $\lambda$. We have to redefine $z_{0}$ and $z_{1}$ in a neighborhood of $\xi_{1}$ and $\xi_{2}$ in a suitable way. So, let $F_{j}: B_{\rho}\left(\xi_{j}\right) \rightarrow N_{0}$ be a diffeomorphism, where $\rho>0$ is fixed and $N_{0}$ is an open neighborhood of $0 \in \mathbb{R}^{2}$ such that

$$
F_{j}\left(\Omega \cap B_{\rho}\left(\xi_{j}\right)\right)=\mathbb{R}_{+}^{2} \cap N_{0}, \quad F_{j}\left(\partial \Omega \cap B_{\rho}\left(\xi_{j}\right)\right)=\partial \mathbb{R}_{+}^{2} \cap N_{0}
$$

and such that $F_{j}$ preserves area.

Copyright (C) by SIAM. Unauthorized reproduction of this article is prohibited. 
For $y \in \Omega_{\lambda} \cap B_{\rho / \lambda}\left(\xi_{j}^{\prime}\right)$ we define

$$
F_{j}^{\lambda}(y)=\frac{1}{\lambda} F_{j}(\lambda y) \quad \text { and } \quad Z_{i j}(y)=z_{i j}\left(F_{j}^{\lambda}(y)\right), \quad j=1,2, \quad i=0,1,
$$

where $z_{i j}$ denotes the function $z_{i}$ with parameter $\mu_{j}$, namely

$$
z_{0 j}=1-2 \mu_{j} \frac{x_{2}+\mu_{j}}{x_{1}^{2}+\left(x_{2}+\mu_{j}\right)^{2}}, \quad z_{1 j}=-2 \frac{x_{1}}{x_{1}^{2}+\left(x_{2}+\mu_{j}\right)^{2}} .
$$

Let $\tilde{\chi}: \mathbb{R} \rightarrow \mathbb{R}$ be a nonnegative smooth function with $\tilde{\chi}(r)=1$ for $r \leq R_{0}$, and let $\tilde{\chi}(r)=0$ for $r \geq R_{0}+1,0 \leq \tilde{\chi} \leq 1$ (with $R_{0}$ a large positive constant). Then, we set

$$
\chi_{j}(y):=\tilde{\chi}\left(\left|F_{j}^{\lambda}(y)\right|\right), j=1,2, \quad \text { and } \quad \chi(y):=\chi_{1}(y)+\chi_{2}(y) .
$$

We will assume that $\lambda$ is small enough to satisfy

$$
\left|F_{j}^{\lambda}(y)\right| \geq R_{0}+1 \quad \forall y \in \Omega_{\lambda} \cap \partial B_{\rho / \lambda}\left(\xi_{j}^{\prime}\right) .
$$

Hence, the products $\chi_{j} Z_{1 j}$ can be defined in the whole domain $\Omega_{\lambda}$ by continuation by zero in $\Omega_{\lambda} \backslash B_{\rho / \lambda}\left(\xi_{j}^{\prime}\right)$. Moreover, by the definition of $Z_{0 j}$ we may also assume that, for fixed $0<b<1$ and suitably chosen $\delta$,

$$
Z_{0 j}(y) \geq 1-\lambda^{b} \quad \forall y \in \Omega_{\lambda} \cap \partial B_{\delta / \lambda}\left(\xi_{j}^{\prime}\right) .
$$

We now define

$$
Z(y)= \begin{cases}\min \left(1-\lambda^{b}, Z_{0 j}(y)\right) & \text { if }\left|y-\xi_{j}^{\prime}\right|<\frac{\delta}{\lambda} \\ 1-\lambda^{b} & \text { if }\left|y-\xi_{j}^{\prime}\right| \geq \frac{\delta}{\lambda} \text { for } j=1,2\end{cases}
$$

We want to solve the following linear problem: given $f \in L^{\infty}\left(\Omega_{\lambda}\right)$ and $h \in$ $L^{\infty}\left(\partial \Omega_{\lambda}\right)$, find $\phi \in L^{\infty}\left(\Omega_{\lambda}\right)$ and $c_{j} \in \mathbb{R}, j=0,1,2$, such that

$$
\left\{\begin{array}{l}
-\Delta_{a_{\lambda}} \phi=f \quad \text { in } \Omega_{\lambda}, \\
\frac{\partial \phi}{\partial \nu}-\mathcal{W} \phi=h+\sum_{j=1}^{2} c_{j} \chi_{j} Z_{1 j}+c_{0} \chi Z \quad \text { on } \partial \Omega_{\lambda} \\
\int_{\Omega_{\lambda}} a \chi Z \phi=0 \text { and } \int_{\Omega_{\lambda}} a \chi_{j} Z_{1 j} \phi=0 \quad \text { for } j=1,2 .
\end{array}\right.
$$

It is necessary to introduce some $L^{\infty}$-weighted norms: if $h \in L^{\infty}\left(\partial \Omega_{\lambda}\right)$ and $f \in L^{\infty}\left(\Omega_{\lambda}\right)$, let

$\|h\|_{*}=\sup _{y \in \partial \Omega_{\lambda}} \frac{|h(y)|}{\sum_{j=1}^{2}\left(1+\left|y-\xi_{j}^{\prime}\right|\right)^{-1-\sigma}} \quad$ and $\quad\|f\|_{* *}=\sup _{y \in \Omega_{\lambda}} \frac{|f(y)|}{\sum_{j=1}^{2}\left(1+\left|y-\xi_{j}^{\prime}\right|\right)^{-2-\sigma}}$,

where $\sigma>0$ is a fixed and small number.

The following result holds.

Proposition 3.5. For any $d>0$, there exist $\lambda_{0}>0$ and $C>0$ such that for any $\lambda \in\left(0, \lambda_{0}\right)$, for any $\xi_{1}, \xi_{2} \in \partial \Omega$ with $\left|\xi_{1}-\xi_{2}\right| \geq d$, for any $h \in L^{\infty}\left(\partial \Omega_{\lambda}\right)$, and 
$f \in L^{\infty}\left(\Omega_{\lambda}\right)$ there is a unique solution $\phi \in L^{\infty}\left(\Omega_{\lambda}\right)$ and $c_{0}, c_{1}, c_{2} \in \mathbb{R}$ to the problem (3.12).

Moreover,

$\|\phi\|_{L^{\infty}\left(\Omega_{\lambda}\right)} \leq C \log \frac{1}{\lambda}\left(\|h\|_{*}+\|f\|_{* *}\right) \quad$ and $\quad \max \left\{\left|c_{0}\right|,\left|c_{1}\right|,\left|c_{2}\right|\right\} \leq C\left(\|h\|_{*}+\|f\|_{* *}\right)$.

Proof. We argue as in the proof of Propositions 1 and 2 of [5]. We only point out

$$
\Delta_{a_{\lambda}} \phi(y)=\Delta \phi(y)+\lambda \frac{\nabla a(\lambda y)}{a(\lambda y)} \phi(y), \quad y \in \Omega / \lambda .
$$

Moreover, the proof exploits a potential theory argument where Green's function for the Laplacian is replaced by Green's function $G_{a}$ whose regular part is studied in Lemma 3.2.

3.3. The nonlinear problem with constraints. In order to solve our problem we need to split the error term $\phi$ in (3.3) as $\phi(y)=\tau Z(y)+\phi_{1}(y)$, where the function $Z$ is defined in (3.11), $\tau=\tau(\lambda)$ is a small parameter, and $\phi_{1}$ satisfies the orthogonal conditions

$$
\int_{\Omega_{\lambda}} a \chi Z \phi_{1}=0 \text { and } \int_{\Omega_{\lambda}} a \chi_{j} Z_{1 j} \phi_{1}=0 \text { for } j=1,2 .
$$

Therefore, the function $v$ in (3.3) reads as

$$
v(y)=V_{1}(y)+\phi_{1}(y), \quad \text { where } V_{1}(y)=V(y)+\tau Z(y), \quad y \in \Omega_{\lambda} .
$$

Moreover, $v$ is a solution for (3.2) if and only if $\phi_{1}$ solves

$$
\begin{cases}-\Delta_{a_{\lambda}} \phi_{1}=\tau \nabla \log a_{\lambda} \cdot \nabla Z & \text { in } \Omega_{\lambda}, \\ \frac{\partial \phi_{1}}{\partial \nu}-\mathcal{W}_{1} \phi_{1}=\mathcal{R}_{1}+\mathcal{N}_{1}\left(\phi_{1}\right) & \text { on } \partial \Omega_{\lambda},\end{cases}
$$

where (see also (3.4), (3.5), and (3.6))

$$
\begin{gathered}
\mathcal{W}_{1}(y):=2 \lambda^{2} \cosh V_{1}(y), \\
\mathcal{R}_{1}(y):=-\left[\frac{\partial V_{1}}{\partial \nu}-2 \lambda^{2} \sinh V_{1}\right](y),
\end{gathered}
$$

and

$$
\mathcal{N}_{1}\left(\phi_{1}\right)=2 \lambda^{2}\left[\sinh \left(V_{1}+\phi_{1}\right)-\sinh V_{1}-\cosh \left(V_{1}\right) \phi_{1}\right] .
$$

It is important to point out that, since $Z(y)=O(1)$ on all $\Omega_{\lambda}$, it follows that $V_{1}(y)=$ $V(y)+O(|\tau|)$ for any $y \in \Omega_{\lambda}$.

Let us consider first the following auxiliary problem: find $\phi_{1} \in L^{\infty}\left(\Omega_{\lambda}\right)$ and $c_{j} \in \mathbb{R}, j=0,1,2$, such that

$$
\left\{\begin{array}{l}
-\Delta_{a_{\lambda}} \phi_{1}=\tau \nabla \log a_{\lambda} \cdot \nabla Z \quad \text { in } \Omega_{\lambda} \\
\frac{\partial \phi_{1}}{\partial \nu}-\mathcal{W}_{1} \phi_{1}=\mathcal{R}_{1}+\mathcal{N}_{1}\left(\phi_{1}\right)+c_{0} \chi Z+c_{1} \chi_{1} Z_{11}+c_{2} \chi_{2} Z_{12} \quad \text { on } \partial \Omega_{\lambda} \\
\int_{\Omega_{\lambda}} a \chi_{j} Z_{1 j} \phi_{1} d x=0 j=1,2, \quad \int_{\Omega_{\lambda}} a \chi Z \phi_{1} d x=0,
\end{array}\right.
$$

Copyright (c) by SIAM. Unauthorized reproduction of this article is prohibited. 
where $\mathcal{W}_{1}, \mathcal{R}_{1}$, and $\mathcal{N}_{1}$ are defined in (3.13), (3.14), and (3.15), respectively.

Lemma 3.6. Let $\alpha \in(0,1), d>0$, and $\tau=O\left(\lambda^{\beta}\right)$ with $\beta>\frac{\alpha}{2}$. Then there are $\lambda_{0}>0$ and $C>0$ such that for any $\lambda \in\left(0, \lambda_{0}\right)$ and for any $\xi_{1}, \xi_{2} \in \partial \Omega$ with $\left|\xi_{1}-\xi_{2}\right| \geq d$, problem (3.16) has a unique solution $\phi_{1} \in L^{\infty}\left(\Omega_{\lambda}\right)$ and $c_{0}, c_{1}, c_{2} \in \mathbb{R}$ such that

$$
\left\|\phi_{1}\right\|_{L^{\infty}\left(\Omega_{\lambda}\right)} \leq C \lambda^{\alpha} .
$$

Furthermore, the function $\left(\tau, \xi_{1}^{\prime}, \xi_{2}^{\prime}\right) \rightarrow \phi_{1}\left(\tau, \xi_{1}^{\prime}, \xi_{2}^{\prime}\right) \in L^{\infty}\left(\Omega_{\lambda}\right)$ is $C^{1}$ and

$$
\left\|D_{\left(\xi_{1}^{\prime}, \xi_{2}^{\prime}\right)} \phi_{1}\right\|_{L^{\infty}\left(\Omega_{\lambda}\right)} \leq C \lambda^{\alpha} \quad \text { and } \quad\left\|D_{\tau} \phi_{1}\right\|_{L^{\infty}\left(\Omega_{\lambda}\right)} \leq C \lambda^{\beta_{1}} \text { for some } \beta_{1}<\beta \text {. }
$$

Proof. We argue as in the proof of Lemma 8 of [5]. The only difference is due to the presence of the right-hand side (RHS) $f=\tau \nabla \log a_{\lambda} \cdot \nabla Z$ in (3.16).

Indeed, first, we point out that

$$
\mathcal{W}_{1}(y)=\mathcal{W}(y)+\underbrace{2 \lambda^{2} \sinh (V) \tau Z+\tau^{2} \lambda^{2} \cosh (V+\bar{\tau} Z) Z^{2}}_{:=\tau B},
$$

where $\mathcal{W}$ is defined in (3.4) and $|\bar{\tau}| \leq|\tau|$. It is easy to check that $\|B\|_{*} \leq C$. Then we write the problem (3.16) in terms of the operator $\mathcal{A}$ that associates to any $\phi_{1} \in$ $L^{\infty}\left(\Omega_{\lambda}\right)$ the unique solution given by Proposition 3.5 with $h=\tau B \phi_{1}+\mathcal{R}_{1}+\mathcal{N}_{1}\left(\phi_{1}\right)$ and $f=\tau \nabla \log a_{\lambda} \cdot \nabla Z$. In terms of $\mathcal{A}$, the problem (3.16) is equivalent to the fixed point problem $\phi_{1}=\mathcal{A}\left(\phi_{1}\right)$. Therefore, we are going to prove that $\mathcal{A}$ is a contraction mapping of the set

$$
\mathcal{C} \equiv\left\{\phi \in C\left(\bar{\Omega}_{\lambda}\right):\|\phi\|_{L^{\infty}\left(\Omega_{\lambda}\right)} \leq \lambda^{\alpha}\right\} .
$$

From Proposition 3.5 we get

$$
\left\|\mathcal{A}\left(\phi_{1}\right)\right\|_{L^{\infty}\left(\Omega_{\lambda}\right)} \leq C|\log \lambda|[\underbrace{|\tau|\left\|B \phi_{1}\right\|_{*}+\left\|\mathcal{N}_{1}\left(\phi_{1}\right)\right\|_{*}+\left\|\mathcal{R}_{1}\right\|_{*}}_{:=\mathcal{D}}+\|f\|_{* *}] .
$$

Arguing as in [5] we get that

$$
\|\mathcal{D}\|_{*} \leq C\left(\lambda^{a-\sigma}+\lambda^{2 \beta} \lambda^{\alpha+\beta}+\lambda^{2 \alpha}\right)
$$

for some $a \in(0,1)$ and $\sigma>0$ small so that $a-\sigma>\alpha$ ( $\sigma$ is the number in the definition of $\|\cdot\|_{*},\|\cdot\|_{* *}$ and $\beta$ is such that $\left.\tau=O\left(\lambda^{\beta}\right)\right)$. On the other hand, it is easy to check that

$$
|f(y)|=O(\lambda \tau|\nabla Z(y)|)=O\left(\lambda^{1+a} \tau \sum_{j=1}^{2}\left(1+\left|y-\xi_{j}^{\prime}\right|\right)^{-1}\right) \text { for any } y \in \Omega_{\lambda},
$$

and so

$$
\|f\|_{* *}=O\left(\tau \lambda^{a-\sigma}\right)=O\left(\lambda^{\beta+a-\sigma}\right) .
$$

Then the proof follows exactly as in Lemma 8 of [5].

Next, we have to choose the parameter $\tau$ so that the nonlinear problem (3.16) has a solution with $c_{0}=0$. This is the result of the next lemma, whose proof can be carried out exactly as the proof of Lemma 9 in [5].

Lemma 3.7. Let $d>0$. For any $\alpha \in(0,1)$, there exist $\lambda_{0}>0$ and $C>0$ such that for $\lambda \in\left(0, \lambda_{0}\right)$, and any $\xi_{1}, \xi_{2} \in \partial \Omega$ with $\left|\xi_{1}-\xi_{2}\right| \geq d$, there exists a unique $\tau$ 
with $|\tau|<C \lambda^{\alpha-b / 2}$ (b is given in (3.11)), such that problem (3.16) admits a unique solution $\phi_{1} \in L^{\infty}\left(\Omega_{\lambda}\right), c_{0}=0$, and $c_{1}, c_{2} \in \mathbb{R}$. Moreover,

$$
\|\phi\|_{L^{\infty}\left(\Omega_{\lambda}\right)} \leq C \lambda^{\alpha}
$$

and the function $\left(\xi_{1}^{\prime}, \xi_{2}^{\prime}\right) \rightarrow \phi_{1}\left(\xi_{1}^{\prime}, \xi_{2}^{\prime}\right)$ is $C^{1}$ and

$$
\left\|D_{\left(\xi_{1}^{\prime}, \xi_{2}^{\prime}\right)} \phi_{1}\right\|_{L^{\infty}\left(\Omega_{\lambda}\right)} \leq C \lambda^{\alpha} .
$$

3.4. The reduced problem and proof of Theorem 1.3. For any $\left(\xi_{1}, \xi_{2}\right) \in$ $\partial \Omega \times \partial \Omega$ with $\xi_{1} \neq \xi_{2}$, we define $\phi\left(\xi_{1}, \xi_{2}\right)$ and $c_{j}\left(\xi_{1}, \xi_{2}\right)$ for $j=1,2$ to be the unique solution to (3.16) with $c_{0}=0$ satisfying (3.17) and (3.18). In this section we shall find the points $\xi_{1}$ and $\xi_{2}$ on the boundary $\partial \Omega$ such that $c_{1}=c_{2}=0$. That choice will provide a solution to our problem.

Lemma 3.8. Let $\alpha \in\left(\frac{1}{2}, \frac{3}{4}\right)$ and $b \in(2(1-\alpha), 1)$ (b is given in (3.11)). It holds true that

$$
c_{i}=\tau \lambda\left[-\frac{\mu_{i}}{2 a\left(\xi_{i}\right)} \nabla_{\partial \Omega} a\left(\xi_{i}\right)+o(1)\right], \quad i=1,2,
$$

uniformly with respect to $\left(\xi_{1}, \xi_{2}\right) \in \partial \Omega \times \partial \Omega$ with $\xi_{1} \neq \xi_{2}$.

Proof. We multiply the first line of (3.16) by $a_{\lambda} \chi_{j} Z_{1 j}, j=1,2$, and we integrate in $y$. We take into account that $V_{1}=V+\tau Z$ and $\tau$ is chosen so that $c_{0}=0$ (see Lemma 3.7). Therefore, we get

$$
\begin{aligned}
\int_{\Omega_{\lambda}} \tau\left(\nabla a_{\lambda} \nabla Z\right) \chi_{j} Z_{1 j} d y= & -\int_{\Omega_{\lambda}} a_{\lambda} \Delta_{a_{\lambda}}\left(\chi_{j} Z_{1 j}\right) \phi_{1} d y \\
& +\int_{\partial \Omega_{\lambda}} a_{\lambda} \phi_{1} \partial_{\nu}\left(\chi_{j} Z_{1 j}\right) d y-\int_{\partial \Omega_{\lambda}} a_{\lambda} \chi_{j} Z_{1 j} \partial_{\nu} \phi_{1} d y
\end{aligned}
$$

and so

$$
\begin{aligned}
& \sum_{i=1}^{2} c_{i} \underbrace{\int_{\partial \Omega_{\lambda}} a(\lambda y) \chi_{i} \chi_{j} Z_{1 i} Z_{1 j} d y}_{I_{0}} \\
& =-\int_{\Omega_{\lambda}} a_{\lambda} \Delta_{a_{\lambda}}\left(\chi_{j} Z_{1 j}\right) \phi_{1} d y-\int_{\Omega_{\lambda}} \tau\left(\nabla a_{\lambda} \nabla Z\right) \chi_{j} Z_{1 j} d y \\
& +\int_{\partial \Omega_{\lambda}} a_{\lambda} \phi_{1} \partial_{\nu}\left(\chi_{j} Z_{1 j}\right) d y-\int_{\partial \Omega_{\lambda}} a_{\lambda} \chi_{j} Z_{1 j}\left(\mathcal{W}_{1} \phi_{1}+\mathcal{R}_{1}+\mathcal{N}_{1}\left(\phi_{1}\right)\right) d y \\
& =-\underbrace{\int_{\Omega_{\lambda}} a_{\lambda} \Delta_{a_{\lambda}}\left(\chi_{j} Z_{1 j}\right) \phi_{1} d y}_{I_{1}}-\underbrace{\int_{\Omega_{\lambda}} \tau\left(\nabla a_{\lambda} \nabla Z\right) \chi_{j} Z_{1 j} d y}_{I_{2}} \\
& +\underbrace{\int_{\partial \Omega_{\lambda}} a_{\lambda} \phi_{1}\left[\partial_{\nu}\left(\chi_{j} Z_{1 j}\right)-\mathcal{W}_{1}\left(\chi_{j} Z_{1 j}\right)\right] d y}_{I_{3}} \\
& +\underbrace{\int_{\partial \Omega_{\lambda}}\left[\frac{\partial V}{\partial \nu}-\lambda^{2} \sinh (V)\right] a_{\lambda} \chi_{j} Z_{1 j} d y}_{I_{4}}+\underbrace{\tau \int_{\partial \Omega_{\lambda}}\left(\frac{\partial Z}{\partial \nu}-\mathcal{W} Z\right) a_{\lambda} \chi_{j} Z_{1 j} d y}_{I_{5}}
\end{aligned}
$$

Copyright (c) by SIAM. Unauthorized reproduction of this article is prohibited. 


$$
\begin{aligned}
& -\underbrace{2 \lambda^{2} \int_{\partial \Omega_{\lambda}} a_{\lambda} \chi_{j} Z_{1 j}[\sinh (V+\tau Z)-\sinh (V)-\tau \cosh (V) Z] d y}_{I_{6}} \\
& -\underbrace{\int_{\partial \Omega_{\lambda}} \mathcal{N}_{1}\left(\phi_{1}\right) a_{\lambda} \chi_{j} Z_{1 j} d y}_{I_{7}} .
\end{aligned}
$$

Now let us estimate each integral $I_{i}$ of (3.19). It is immediate to check that

$$
I_{0}=\frac{2 \pi}{\mu_{i}} a\left(\xi_{i}\right)+o(1) \text { if } i=j \quad \text { and } \quad I_{0}=0 \text { if } i \neq j .
$$

Indeed if $i=j$,

$$
\int_{\partial \Omega_{\lambda}} a(\lambda y) \chi_{i} \chi_{j} Z_{1 i} Z_{1 j} d y=a\left(\xi_{i}\right) \int_{\mathbb{R}} \frac{4 x_{1}^{2}}{\left(x_{1}^{2}+\mu_{i}^{2}\right)^{2}} d x_{1}+o(1)=\frac{2 \pi a\left(\xi_{i}\right)}{\mu_{i}}+o(1) .
$$

We remark that

$$
Z_{1 j}=O\left(\frac{1}{1+r}\right) \quad \text { and } \quad \nabla Z_{1 j}=O\left(\frac{1}{1+r^{2}}\right) \text { as } r \rightarrow \infty .
$$

We have

$$
\begin{aligned}
a_{\lambda} \Delta_{a_{\lambda}}\left(\chi_{j} Z_{1 j}\right) & =\Delta \chi_{j} \cdot Z_{1 j}+2 \nabla \chi_{j} \nabla Z_{1 j}+\chi_{j} \Delta Z_{1 j}+\nabla a_{\lambda} \nabla \chi_{j} Z_{1 j}+\chi_{j} \nabla a_{\lambda} \nabla Z_{1 j} \\
& =O\left(\frac{\lambda^{2}}{1+r}\right)+O\left(\frac{\lambda}{1+r^{2}}\right)+\chi_{j} \Delta Z_{1 j}+O\left(\frac{\lambda^{2}}{1+r}\right)+O\left(\frac{\lambda}{1+r^{2}}\right) .
\end{aligned}
$$

However,

$$
\Delta_{y} Z_{1 j}=\Delta_{x} z_{1}+O\left(\lambda|x|\left|\nabla^{2} z_{1}\right|\right)+O\left(\lambda\left|\nabla z_{1}\right|\right),
$$

and hence

$$
\Delta Z_{1 j}=O\left(\frac{\lambda}{1+r^{2}}\right)+O\left(\frac{\lambda^{2}}{1+r}\right)
$$

Hence

$$
\left|I_{1}\right| \leq C \lambda\|\phi\|_{L^{\infty}\left(\Omega_{\lambda}\right)} \leq C \lambda^{1+\alpha}=o\left(\lambda^{1+\alpha-\frac{b}{2}}\right) .
$$

Moreover,

$$
\begin{aligned}
\left|I_{3}\right| & \leq\left|\int_{\partial \Omega_{\lambda}} a_{\lambda} \phi_{1} \partial_{\nu}\left(\chi_{j}\right) Z_{1 j} d y\right|+\left|\int_{\partial \Omega_{\lambda}} a_{\lambda} \phi_{1} \chi_{j}\left(\partial_{\nu} Z_{1 j}-\mathcal{W}_{1} Z_{1 j}\right) d y\right| \\
& \leq C \lambda\left\|\phi_{1}\right\|_{L^{\infty}\left(\Omega_{\lambda}\right)} \log \frac{1}{\lambda}+C \lambda^{\alpha}\left\|\phi_{1}\right\|_{L^{\infty}\left(\Omega_{\lambda}\right)} \log \frac{1}{\lambda} \\
& \leq C\left(\lambda^{1+\alpha} \log \frac{1}{\lambda}+\lambda^{2 \alpha} \log \frac{1}{\lambda}\right)=o\left(\lambda^{1+\alpha-\frac{b}{2}}\right),
\end{aligned}
$$

because $\nabla \chi_{j}=O(\lambda)$ and (see also (3.39) in [5])

$$
\chi_{j}\left(\partial_{\nu} Z_{1 j}-\mathcal{W}_{1} Z_{1 j}\right)=O\left(\frac{\lambda^{\alpha}}{1+\left|y-\xi_{j}^{\prime}\right|}\right)
$$

Copyright (c) by SIAM. Unauthorized reproduction of this article is prohibited. 
and since $b>2(1-\alpha)$.

By (3.8) we get that

$$
\mathcal{R}=\left(z_{\lambda}^{\xi_{1}}(\lambda y)-z_{\lambda}^{\xi_{2}}(\lambda y)\right) e^{H_{a}\left(\lambda y, \xi_{j}\right)-H_{a}\left(\xi_{j}, \xi_{j}\right)}+O\left(\lambda^{2}\right),
$$

and hence by making the change of variable $x=F_{j}^{\lambda}(y)$ and by observing that $\left(F_{j}^{\lambda}\right)^{-1}(x)=x+\xi_{j}^{\prime}+O(\lambda|x|)$ we get

$$
\begin{aligned}
I_{4}= & 2 \mu_{j} \int_{-\frac{\rho}{\lambda}}^{\frac{\rho}{\lambda}} z_{\lambda}^{\xi_{j}}\left(\lambda x+\xi_{j}\right) a\left(\lambda x+\xi_{j}\right) e^{\lambda x+O\left(\lambda^{2}|x|^{2}\right)} \partial_{x_{1}} \frac{1}{x_{1}^{2}+\mu_{j}^{2}} \\
= & 2 \mu_{j} \int_{-\frac{\rho}{\lambda}}^{\frac{\rho}{\lambda}} \partial_{x_{1}} z_{\lambda}^{\xi_{j}}\left(\lambda x+\xi_{j}\right) a\left(\lambda x+\xi_{j}\right) e^{\lambda x+O\left(\lambda^{2}|x|^{2}\right)} \frac{1}{x_{1}^{2}+\mu_{j}^{2}} \\
& -2 \mu_{j} \lambda \int_{-\frac{\rho}{\lambda}}^{\frac{\rho}{\lambda}} \partial_{x_{1}} a\left(\xi_{j}+\lambda x+O\left(\lambda^{2}|x|\right)\right) z_{\lambda}^{\xi_{j}}\left(\lambda x+\xi_{j}\right) e^{\lambda x+O\left(\lambda^{2}|x|^{2}\right)} \frac{1}{x_{1}^{2}+\mu_{j}^{2}} \\
& -2 \mu_{j} \lambda \int_{-\frac{\rho}{\lambda}}^{\frac{\rho}{\lambda}} \partial_{x_{1}} z_{\lambda}^{\xi_{j}}\left(\xi_{j}+\lambda x+O\left(\lambda^{2}|x|\right)\right) a\left(\lambda x+\xi_{j}\right) e^{\lambda x+O\left(\lambda^{2}|x|^{2}\right)} \frac{1}{x_{1}^{2}+\mu_{j}^{2}} \\
& -2 \mu_{j} \lambda \int_{-\frac{\rho}{\lambda}}^{\frac{\rho}{\lambda}} z_{\lambda}^{\xi_{j}}\left(\xi_{j}+\lambda x+O\left(\lambda^{2}|x|\right)\right) a\left(\lambda x+\xi_{j}\right) \partial_{x_{1}} e^{\lambda x+O\left(\lambda^{2}|x|^{2}\right)} \frac{1}{x_{1}^{2}+\mu_{j}^{2}}
\end{aligned}
$$

and so

$$
\left|I_{4}\right| \leq \lambda^{1+\alpha} \log \frac{1}{\lambda}=o\left(\lambda^{1+\alpha-\frac{b}{2}}\right) .
$$

As in the estimates proved on p. 211 of [5], we get that

$$
\chi_{j}\left(\partial_{\nu} Z-\mathcal{W} Z\right)=\left(z_{\lambda}^{\xi_{1}}(\lambda y)-z_{\lambda}^{\xi_{2}}(\lambda y)\right) e^{H_{a}\left(\lambda y, \xi_{j}\right)-H_{a}\left(\xi_{j}, \xi_{j}\right)}+O\left(\lambda^{2}\right),
$$

and so, by making computations as before we get that

$$
\left|I_{5}\right| \leq C \lambda^{1+\alpha} \tau \log \frac{1}{\lambda}=o\left(\lambda^{1+\alpha-\frac{b}{2}}\right) .
$$

Moreover, since by the mean value theorem

$$
\chi_{j} \lambda^{2}[\sinh (V+\tau Z)-\sinh (V)-\tau \cosh (V) Z]=\chi_{j} \tau^{2} \sinh (V+\bar{\tau} Z) Z^{2},
$$

by making again the same computations as before we also have

$$
\left|I_{6}\right| \leq C \lambda \tau^{2} \log \frac{1}{\lambda}=o\left(\lambda^{1+\alpha-\frac{b}{2}}\right) .
$$

Finally, we have

$$
\left|I_{7}\right| \leq C\|\phi\|_{L^{\infty}\left(\Omega_{\lambda}\right)}^{2} \log \frac{1}{\lambda} \leq C \lambda^{2 \alpha} \log \frac{1}{\lambda} .
$$

Therefore, it remains to estimate the leading term $I_{2}$ of the RHS of (3.19). We observe that in the function $Z$ is constant except in the regions $\left|y-\xi_{j}^{\prime}\right|<\mu_{j} \lambda^{-b / 2}$, $j=1,2$. For the sake of simplicity, we can also assume that the boundary of $\Omega$ in 
a neighborhood of the point $\xi_{j}$ can be described as a graph of a smooth function $\varphi_{j}$ defined in a neighborhood of 0 such that $\varphi_{j}(0)=\varphi_{j}^{\prime}(0)=0$ so that

$$
F_{j}\left(s_{1}, s_{2}\right)=\left(s_{1}, s_{2}-\varphi_{j}\left(s_{1}\right)\right) \quad \text { and } \quad F_{j}^{-1}\left(t_{1}, t_{2}\right)=\left(t_{1}, t_{2}+\varphi_{j}\left(t_{1}\right)\right) .
$$

Let us remember that $F_{j}^{\lambda}(y)=\frac{F_{j}(\lambda y)}{\lambda}$; then

$$
\begin{aligned}
& I_{2}=\int_{\Omega_{\lambda}} \tau\left(\nabla a_{\lambda}(y) \nabla Z(y)\right) \chi_{j}(y) Z_{1 j}(y) d y \\
& =\tau \int_{\Omega_{\lambda}} \nabla a(\lambda y) \nabla\left(z_{0 j}\left(F_{j}^{\lambda}(y)\right)\right) \tilde{\chi}\left(\left|F_{j}^{\lambda}(y)\right|\right) z_{1 j}\left(F_{j}^{\lambda}(y)\right) d y \\
& =\tau \int_{\Omega_{\lambda}}\left\{\frac{\partial a_{\lambda}}{\partial y_{1}}\left[\frac{\partial z_{0 j}}{\partial x_{1}} \frac{\partial\left(F_{j}^{\lambda}\right)_{1}}{\partial y_{1}}+\frac{\partial z_{0 j}}{\partial x_{2}} \frac{\partial\left(F_{j}^{\lambda}\right)_{2}}{\partial y_{1}}\right]+\frac{\partial a_{\lambda}}{\partial y_{2}}\left[\frac{\partial z_{0 j}}{\partial x_{1}} \frac{\partial\left(F_{j}^{\lambda}\right)_{1}}{\partial y_{2}}+\frac{\partial z_{0 j}}{\partial x_{2}} \frac{\partial\left(F_{j}^{\lambda}\right)_{2}}{\partial y_{2}}\right]\right\} \\
& \quad \times \tilde{\chi}\left(\left|F_{j}^{\lambda}(y)\right|\right) z_{1 j}\left(F_{j}^{\lambda}(y)\right) d y \\
& =\tau \lambda \int_{\Omega_{\lambda}}\left\{\frac{\partial a}{\partial y_{1}}(\lambda y)\right. \\
& \left.\quad\left[\frac{\partial z_{0 j}}{\partial x_{1}}\left(F_{j}^{\lambda}(y)\right)-\frac{\partial z_{0 j}}{\partial x_{2}}\left(F_{j}^{\lambda}(y)\right) \varphi_{j}^{\prime}\left(\lambda y_{1}\right)\right]+\frac{\partial a}{\partial y_{2}}(\lambda y) \frac{\partial z_{0 j}}{\partial x_{2}}\left(F_{j}^{\lambda}(y)\right)\right\} \\
& \times \tilde{\chi}\left(\left|F_{j}^{\lambda}(y)\right|\right) z_{1 j}\left(F_{j}^{\lambda}(y)\right) d y
\end{aligned}
$$

(we set $F_{j}^{\lambda}(y)=x$, i.e., $y=\frac{F_{j}^{-1}(\lambda x)}{\lambda}$ )

$=\tau \lambda \int_{B(0, \rho) \cap \mathbb{R}_{+}^{2}}\left\{\frac{\partial a}{\partial y_{1}}\left(\lambda x_{1}, \lambda x_{2}+\varphi_{j}\left(\lambda x_{1}\right)\right)\left[\frac{\partial z_{0 j}}{\partial x_{1}}(x)-\frac{\partial z_{0 j}}{\partial x_{2}}(x) \varphi_{j}^{\prime}\left(\lambda x_{1}\right)\right]\right\}$

$$
+\tau \lambda \int_{B(0, \rho) \cap \mathbb{R}_{+}^{2}}\left\{\frac{\partial a}{\partial y_{2}}\left(\lambda x_{1}, \lambda x_{2}+\varphi_{j}\left(\lambda x_{1}\right)\right) \frac{\partial z_{0 j}}{\partial x_{2}}(x)\right\} \times \tilde{\chi}(|x|) z_{1 j}(x) d x
$$$$
=\tau \lambda\left(\frac{\partial a}{\partial y_{1}}(0) \int_{\mathbb{R}_{+}^{2}} \frac{\partial z_{0 j}}{\partial x_{1}}(x) z_{1 j}(x) d x+o(1)\right)
$$$$
=\tau \lambda\left(\frac{\partial a}{\partial y_{1}}(0) \int_{\mathbb{R}_{+}^{2}}\left(-8 \mu_{j} \frac{x_{1}^{2}\left(x_{2}+\mu_{j}\right)}{\left(x_{1}^{2}+\left(x_{2}+\mu_{j}\right)^{2}\right)^{3}}\right) d x+o(1)\right)
$$$$
=\tau \lambda\left(-\pi \nabla_{\partial \Omega} a\left(\xi_{j}\right)+o(1)\right) \text {. }
$$

We point out that this is the lower order term of the RHS of (3.19), because its rate is of order $\lambda^{1+\alpha-b / 2}$ because of the choice of $\tau$.

The claim follows collecting all of the previous estimates.

Finally, we conclude with the proof of Theorem 1.3.

Proof (Theorem 1.3 completed). Let $\xi_{1}$ and $\xi_{2}$ be two different $C^{1}$-stable critical points of $a$ restricted on $\partial \Omega$, namely the local Brouwer degree

$$
\operatorname{deg}\left(\nabla_{\partial \Omega} a, B\left(\xi_{i}, \rho\right) \cap \partial \Omega, 0\right) \neq 0 \quad \text { for } i=1,2
$$

provided $\rho$ is small enough. Then by the product property we deduce

$$
\operatorname{deg}\left(\left(\nabla_{\partial \Omega} a, \nabla_{\partial \Omega} a\right),\left(B\left(\xi_{1}, \rho\right) \times B\left(\xi_{2}, \rho\right)\right) \cap(\partial \Omega \times \partial \Omega), 0\right) \neq 0,
$$

which implies together with Lemma 3.8 that if $\lambda$ is small enough, there exists $\left(\xi_{1}^{\lambda}, \xi_{2}^{\lambda}\right)$ which approaches $\left(\xi_{1}, \xi_{2}\right)$ as $\lambda$ goes to zero such that $c_{1}=c_{2}=0$. Therefore, $v_{\lambda}(y)=$ 
$V(y)+\phi(y), y \in \Omega_{\lambda}$, turns out to be a solution to the problem (3.2). It is clear that the scaled function $u_{\lambda}(x)=v_{\lambda}(x / \lambda), x \in \Omega$, is the solution to problem (1.7), which satisfies (1.12) and concentrates at the points $\xi_{1}$ and $\xi_{2}$ as $\lambda$ goes to zero.

\section{REFERENCES}

[1] P. Bartolo, V. Benci, And D. Fortunato, Abstract critical point theorems and applications to some nonlinear problems with "strong" resonance at infinity, Nonlinear Anal., 7 (1983), pp. 981-1012.

[2] K. BRYAn AND M. Vogelius, Singular solutions to a nonlinear elliptic boundary value problem originating from corrosion modelling, Quart. Appl. Math., 60 (2002), pp. 675-694.

[3] P. Cherrier, Problèmes de Neumann nonlinéaires sur les variétés riemanniennes, J. Funct. Anal., 57 (1984), pp. 154-206.

[4] J. Davila, M. Del Pino, and M. Musso, Concentrating solutions in a two-dimensional elliptic problem with exponential Neumann data, J. Funct. Anal., 227 (2005), pp. 430-490.

[5] J. Davila, M. del Pino, M. Musso, And J. Wei, Singular limits of a two dimensional boundary value problem arising in corrosion modelling, Arch. Ration. Mech. Anal., 182 (2006), pp. $181-221$.

[6] O. KaVian and M. Vogelius, On the existence and 'blow-up' of solutions to a two-dimensional nonlinear boundary-value problem arising in corrosion modelling, Proc. Roy. Soc. Edinburgh Sect. A, 133 (2003), pp. 119-149, corrigendum to the same, Proc. Roy. Soc. Edinburgh Sect. A, 133 (2003), pp. 729-730.

[7] C.D. Pagani And D. Pierotti, Variational methods for nonlinear Steklov eigenvalues problems with an indefinite weight function, Calc. Var. Partial Differential Equations, 39 (2010), pp. $35-58$.

[8] C.D. Pagani And D. Pierotti, Multiple variational solutions to non linear Steklov problems, NoDEA Nonlinear Differential Equations Appl., 19 (2012), pp. 417-436.

[9] C.D. Pagani And D. Pierotti, A three dimensional Steklov eigenvalue problem with exponential nonlinearity on the boundary, Nonlinear Anal., 79 (2013), pp. 28-40.

[10] C.D. Pagani, D. Pierotti, G.M. Verzini, And A. Zilio, A nonlinear Steklov problem arising in corrosion modelling, in Contributions to Nonlinear Elliptic Equations and Systems, Progress in Nonlinear Differential Equations and Their Applications 86, Birkhäuser Boston, Boston, 2015, pp. 371-387.

[11] M. Struwe, Variational Methods and Applications to Nonlinear Partial Differential Equations and Hamiltonian Systems, Springer-Verlag, Berlin, 1990.

[12] M. Vogelius And J.-M. XU, A nonlinear elliptic boundary value problem related to corrosion modelling, Quart. Appl. Math., 56 (1998), pp. 479-505.

[13] J. WeI, D. YE, AND F. Zhou, Bubbling solutions for an anisotropic Emden-Fowler equation, Calc. Var. Partial Differential Equations, 28 (2007), pp. 217-247.

Copyright (c) by SIAM. Unauthorized reproduction of this article is prohibited. 